MATHEMATICS OF COMPUTATION

Volume 66, Number 218, April 1997, Pages 495-526

S 0025-5718(97)00822-3

\title{
VISCOSITY METHODS FOR PIECEWISE SMOOTH SOLUTIONS TO SCALAR CONSERVATION LAWS
}

\author{
TAO TANG AND ZHEN-HUAN TENG
}

\begin{abstract}
It is proved that for scalar conservation laws, if the flux function is strictly convex, and if the entropy solution is piecewise smooth with finitely many discontinuities (which includes initial central rarefaction waves, initial shocks, possible spontaneous formation of shocks in a future time and interactions of all these patterns), then the error of viscosity solution to the inviscid solution is bounded by $O(\epsilon|\log \epsilon|+\epsilon)$ in the $L^{1}$-norm, which is an improvement of the $O(\sqrt{\epsilon})$ upper bound. If neither central rarefaction waves nor spontaneous shocks occur, the error bound is improved to $O(\epsilon)$.
\end{abstract}

\section{INTRODUCTION}

Consider the single hyperbolic conservation laws

$$
u_{t}+f(u)_{x}=0, \quad-\infty<x<\infty, \quad t>0,
$$

subject to the initial condition

$$
u(x, 0)=u_{0}(x), \quad-\infty<x<\infty .
$$

The viscosity method approximating the conservation laws (0.1) and (0.2) is to solve the parabolic equation

$$
\left(v_{\epsilon}\right)_{t}+f\left(v_{\epsilon}\right)_{x}=\epsilon\left(v_{\epsilon}\right)_{x x}
$$

subject to the same initial condition

$$
v_{\epsilon}(x, 0)=u_{0}(x) .
$$

Here, $\epsilon>0$ is a small parameter. Viscosity methods play an important role in theoretical analysis on numerical methods for hyperbolic conservation laws. A useful technique for studying the behavior of solutions to difference equations is to model the difference equation by a differential equation called modified equation, see for example Chapter 11 of LeVeque's book [13]. The difference equation was introduced because it is easier to solve numerically than the partial differential equation (PDE). This is true if we want to generate numerical approximations, but on the other hand it is often easier to predict qualitative behavior of a PDE than of a system of difference equations. Since many modified equations have similar

Received by the editor November 2, 1995 and, in revised form, April 5, 1996.

1991 Mathematics Subject Classification. Primary 65M10, 65M05, 35L65.

Key words and phrases. Hyperbolic conservation laws, error estimate, viscosity methods, piecewise smooth.

Research of the first author was supported by NSERC Canada Grant OGP0105545.

Research of the second author was supported by the National Natural Science Foundation of China and the Science Fund of the Education Commission of China.

(C)1997 American Mathematical Society 
forms to equation (0.3) (more precise form is equation (6.1), see Section 6 for more discussions), a better understanding of the solution behavior of (0.3) will help us to understand many difference methods for (0.1). For existence and regularity properties for $(0.3)$, we refer to $[9,16]$.

In this work, we are concerned with the accuracy of the viscosity methods. More precisely, we will investigate the $L^{1}$-convergence rate of $u-v_{\epsilon}$. It is well known that the $L^{1}$-norm of error decays only like $O(\sqrt{\epsilon})$ if the initial data is piecewise smooth. Consider an advection diffusion equation of the form

$$
v_{t}+a v_{x}=\epsilon v_{x x}, \quad a=\text { constant },
$$

with $u_{0}(x)=-\operatorname{sign}(x)$. Its exact solution is

$$
v_{\epsilon}(x, t)=1-\frac{4}{\sqrt{\pi}} \int_{-\infty}^{(x-a t) / \sqrt{4 \epsilon t}} e^{-y^{2}} d y .
$$

From this and the fact that the solution to the pure advection problem is $u(x, t)=$ $-\operatorname{sign}(x-a t)$, it can be shown that

$$
\left\|u(\bullet, t)-v_{\epsilon}(\bullet, t)\right\|_{L^{1}(\mathbf{R})}=C \sqrt{\epsilon t}
$$

for some constant $C$ independent of $\epsilon$ and $t$. This indicates that the $L^{1}$-convergence rate of the viscosity methods is only one half for this simple linear problem. In practice, solutions of monotone difference methods agree very well with the true solutions of related modified equations. It is expected that monotone difference methods will have similar convergence rate to that of viscosity methods. Harten, Hyman and Lax [7] pointed out that monotone difference methods are of at most first-order accuracy and Kuznetsov [10] provided an one half order $L^{1}$-convergence rate for BV bounded initial data. Tang and Teng [19] recently proved that the best $L^{1}$-convergence rate for monotone difference methods to scalar conservation laws is one half if it includes the linear flux case. All these indicate that viscosity methods and monotone difference methods share some common properties on the $L^{1}$-convergence rate.

Although it is proved that half order convergence rate is best possible for both viscosity methods and monotone difference methods, it is widely believed that these methods may have higher order of convergence rate when applying to convex conservation laws. More precisely, if the flux $f$ is strictly convex,

$$
f^{\prime \prime} \geq \alpha>0,
$$

then higher order (greater than one half) convergence rate is expected, see Harten [6]. Lucier [14] used one of the monotone schemes, Godunov's method, to solve Riemann problems for (0.1) with a convex flux. He observed an order one convergence rate. Teng and Zhang [20] recently proved that for a special class of initial data, namely piecewise decreasing constants, both viscosity and strictly monotone difference methods have order one $L^{1}$-convergence rate, provided that the flux $f$ is strictly convex. Likewise, Bakhvalov [1] and Harabetian [5] proved a faster convergence rate $O(\epsilon|\ln \epsilon|)$ for Riemann problems with a rarefaction wave. Teng and Zhang's work used the existence of traveling waves obtained by Jennings [8]. It is noted that Jennings' results do not cover Godunov's method since his proof relies heavily on the strict monotonicity of the schemes and differentiability of the flux function. In a recent work of Fan [3], the existence and structure of traveling 
waves for Godunov's method are investigated. The order one $L^{1}$-convergence rate is established for Godunov's method on single shock solutions.

In this paper, we also consider (0.1) with a strictly convex flux, but with a more general and practical class of initial data. With this class of initial data, the entropy solution to (0.1) may include initial central rarefaction waves, initial shocks, possible spontaneous formation of shocks in a future time and interactions of all these patterns. We will establish $O(\epsilon|\ln \epsilon|) L^{1}$-convergence rate for the piecewise smooth solutions with finitely many discontinuities. To the best of our knowledge, no global error estimates for piecewise smooth solutions have been obtained, although some results for Riemann problems [1, 3, 5], for piecewise constant solutions [20], and for BV bounded initial data $[14,10]$ are available. For piecewise smooth solutions to hyperbolic systems with finitely many noninteracting shocks, $O(\epsilon)$ local error estimates have been obtained by Goodman and Xin [4].

The remainder of the paper consists of six sections. In Section 1, we investigate the structures of the solution to (0.1) with a class of piecewise smooth initial data. Some useful estimates for first order and second order derivatives of the solution are obtained. In Section 2, we introduce a traveling wave solution and a stability lemma, which play important roles in obtaining the $L^{1}$-convergence rate. Using the stability lemma, we prove in Section 3 the following estimate:

$$
\sup _{0 \leq t \leq T}\left\|u(\bullet, t)-v_{\epsilon}(\bullet, t)\right\|_{L^{1}(\mathbf{R})} \leq C(T)(\epsilon|\log \epsilon|+\epsilon),
$$

provided that $u_{0}$ is piecewise smooth and that $f^{\prime}\left(u_{0}\right)$ has a finite number of inflection points. In our proof, we will use a matching method developed by Goodman and Xin [4] and Liu and Xin [12]. In Section 4, we make some remarks on optimal error estimates. It is shown that if neither initial central rarefaction waves nor spontaneous shocks occur, then

$$
\sup _{0 \leq t \leq T}\left\|u(\bullet, t)-v_{\epsilon}(\bullet, t)\right\|_{L^{1}(\mathbf{R})} \leq C(T) \epsilon .
$$

In Section 5, we derive sharp estimates for viscosity methods with a special class of initial function, which includes the initial data considered in Teng and Zhang [20]. Loosely speaking, we consider a nonincreasing piecewise smooth solution with finitely many shock interactions. In this case, we obtain the following estimate:

$$
\sup _{0 \leq t<\infty}\left\|u(\bullet, t)-v_{\epsilon}(\bullet, t)\right\|_{L^{1}(\mathbf{R})} \leq c \epsilon,
$$

for some $c$ independent of $\epsilon$. Some possible generalizations will be discussed in the final section.

\section{Solution structures}

Throughout this paper, the norm $\|\cdot\|$ denotes the standard $L^{1}$-norm, $\|\cdot\|_{L^{1}(\mathbf{R})}$; $C$ denotes a positive constant independent of $\epsilon ; c$ denotes a positive constant independent of $t$ and $\epsilon$, but with different values at different places.

Let $a(u)=f^{\prime}(u)$. If $x=x^{*}$ is an inflection point of $a\left(u_{0}(x)\right)$ and also satisfies $\dot{a}\left(u_{0}\left(x^{*}\right)\right)<0$, then we call $x^{*}$ a decreasing inflection point. If this inflection point satisfies $\dot{a}\left(u_{0}\left(x^{*}\right)\right) \geq 0$, we call it an increasing inflection point. Throughout this 
paper, we use the following notations:

$$
\begin{aligned}
& \dot{a}\left(u_{0}(x)\right):=\frac{d}{d x}\left(a\left(u_{0}(x)\right)\right) ; \quad \ddot{a}\left(u_{0}(x)\right):=\frac{d^{2}}{d x^{2}}\left(a\left(u_{0}(x)\right)\right) ; \\
& \dot{u}_{0}(x):=\frac{d u_{0}(x)}{d x} ; \quad \ddot{u}_{0}(x):=\frac{d^{2} u_{0}(x)}{d x^{2}} .
\end{aligned}
$$

The behavior and structure of entropy solutions for scalar convex conservation laws have been studied for many years, see for example $[2,11,15,17]$. It is well known that if the initial function is piecewise $C^{2}$-smooth, then the entropy solutions consist of at most a countable number of $C^{2}$-smooth regions. Tadmor and Tassa [18] proved that if the initial speed has a finite number of decreasing inflection points, then it bounds the number of future shock discontinuities.

We make the following assumptions on the initial data:

- (A1): $u_{0}(x)$ is bounded and piecewise $C^{2}$-smooth with a finite number of discontinuous points $\gamma_{i}, 1 \leq i \leq I ; u_{0}\left(\gamma_{i} \pm 0\right)$ and $\dot{u}_{0}\left(\gamma_{i} \pm 0\right)$ exist and are finite;

- (A2): $\ddot{u}_{0} \in L^{1}(\mathbf{R})$;

- (A3):

$$
\lim _{|x| \rightarrow \infty} \dot{a}\left(u_{0}(x)\right)=0
$$

- (A4): $\ddot{a}\left(u_{0}(x)\right)$ changes signs a finite number of times, i.e. $a\left(u_{0}(x)\right)$ has a finite number of inflection points.

Remark 1.1. In order to obtain an estimate for $u_{x x}$ (Lemma 1.3), we have to assume that $u_{0}$ is piecewise $C^{2}$. The only place to use this assumption is to obtain the estimate (1.30). Otherwise, a weak assumption on $u_{0}$, i.e. $u_{0}$ is piecewise $C^{1}$, will be sufficient. Furthermore, the existence of $\ddot{u}_{0}\left(\gamma_{i} \pm 0\right)$ is not required. That is, $\ddot{u}_{0}\left(\gamma_{i} \pm 0\right)$ can be infinity.

We now introduce following notations:

- Denote the set of points where $\dot{a}\left(u_{0}\right)$ has a negative minimum by $\zeta_{p}, 1 \leq p \leq$ $P$. Without loss of generality, we assume that $\dot{a}\left(u_{0}\left(\zeta_{p}\right)\right)$ are distinct:

$$
\dot{a}\left(u_{0}\left(\zeta_{1}\right)\right)<\dot{a}\left(u_{0}\left(\zeta_{2}\right)\right)<\cdots<\dot{a}\left(u_{0}\left(\zeta_{P}\right)\right) .
$$

It is straightforward to extend our results to the case when there are some $\zeta_{p}, \zeta_{q}, p \neq q$, such that $\dot{a}\left(u_{0}\left(\zeta_{p}\right)\right)=\dot{a}\left(u_{0}\left(\zeta_{q}\right)\right)$.

- Denote $t_{0}=0$ and $t_{p}=-1 / \dot{a}\left(u_{0}\left(\zeta_{p}\right)\right), p=1, \cdots, P$. For a fixed $T>t_{P}$, we let $t_{P+1}=T$.

Remark 1.2. A negative minimum point may form a shock at a future time. However, by no means will every negative minimal point eventually generate a new shock. It is an easier case if the negative minimal point does not lead to a shock. Without loss of generality, we assume that each point in the set $\left\{\zeta_{p}\right\}$ corresponds to a new shock created at a later time. The starting point of the new shock is $\left(x_{p}, t_{p}\right)$.

Since $u_{0}$ is assumed to be only piecewise $C^{2}$, it may be discontinuous and therefore will not have a classical derivative. We refer by $\dot{a}\left(u_{0}\right)$ to the generalized derivative of $a\left(u_{0}\right)$. In the smooth interval $\left(\gamma_{i}, \gamma_{i+1}\right), \dot{a}\left(u_{0}\right)$ is the classical derivative. In positive and negative jump points of $u_{0}, \dot{a}\left(u_{0}\right)$ is positive and negative infinity, respectively. In particular, in negative jump points (i.e. decreasing discontinuities) 
of $u_{0}, \dot{a}\left(u_{0}\right)$ has a negative (infinite) minimum. If $\dot{a}\left(u_{0}\right)$ has a continuum of negative minimal points it is considered as one minimum.

The assumption (A4) indicates that $\dot{a}\left(u_{0}\right)$ has a finite number of decreasing inflection points. It can be shown that under the assumptions (A1), (A3) and (A4) only a finite number of shocks will occur (see, e.g. [17, 18]). The corresponding entropy solution consists of a finite number of $C^{2}$-smooth pieces. The shocks are disjoint, except for common endpoints arising from collisions, and (without loss of generality) we assume that no more than two shocks ever collide. More precisely, for each time interval $\left[t_{p}, t_{p+1}\right]$ there exist a finite number of subintervals, $t^{(p, n)} \leq$ $t<t^{(p, n+1)}, n=0,1, \cdots, N_{p}-1$, where $t^{(p, 0)}=t_{p}, t^{\left(p, N_{p}\right)}=t_{p+1}$, such that in each interval $\left[t^{(p, n)}, t^{(p, n+1)}\right)$ there are a finite number of smooth curves $x=$ $X_{m}^{(p, n)}(t), m=1, \cdots, M_{p, n}$, satisfying

1. For $t \in\left(t^{(p, n)}, t^{(p, n+1)}\right)$,

$$
X_{m}^{(p, n)}(t)<X_{m+1}^{(p, n)}(t), \quad m=1, \cdots, M_{p, n}-1 ;
$$

2. there exists at least one $m$ such that

$$
X_{m}^{(p, n)}\left(t^{(p, n+1)}\right)=X_{m+1}^{(p, n)}\left(t^{(p, n+1)}\right) ;
$$

3. $u(x, t)$ is smooth except on $x=X_{m}^{(p, n)}(t), \quad t^{(p, n)} \leq t<t^{(p, n+1)}$;

4. $u\left(X_{m}^{(p, n)} \pm 0, t\right)$ exist and satisfy either $u\left(X_{m}^{(p, n)}-0, t\right)>u\left(X_{m}^{(p, n)}+0, t\right)$, i.e. $X_{m}^{(p, n)}$ is a shock, or $\left(X_{m}^{(p, n)}-0, t\right)=u\left(X_{m}^{(p, n)}+0, t\right)$, i.e. $X_{m}^{(p, n)}$ is a contact discontinuity. Moreover, for $t \in\left(t^{(p, n)}, t^{(p, n+1)}\right)$,

$$
\dot{X}_{m}^{(p, n)}(t)=\left\{\begin{array}{c}
\frac{f\left(u\left(X_{m}^{(p, n)}+0, t\right)\right)-f\left(u\left(X_{m}^{(p, n)}-0, t\right)\right)}{u\left(X_{m}^{(p, n)}+0, t\right)-u\left(X_{m}^{(p, n)}-0, t\right)}, \\
\text { if } u\left(X_{m}^{(p, n)}+0, t\right)>u\left(X_{m}^{(p, n)}-0, t\right), \\
f^{\prime}\left(u\left(X_{m}^{(p, n)}, t\right)\right), \\
\quad \text { if } u\left(X_{m}^{(p, n)}+0, t\right)=u\left(X_{m}^{(p, n)}-0, t\right),
\end{array}\right.
$$

where $\dot{X}(t)=d X(t) / d t$

5. Each shock $X_{m}^{(p, n)}(t)$ continues to the next subinterval $\left[t^{(p, n+1)}, t^{(p, n+2)}\right)$, with a possibility that it collides with another wave at $t=t^{(p, n+1)}$. Consequently, each shock curve can be extended to $t=\infty$.

From the above results it follows that the solution of (0.1) and (0.2) is a finite combination of the cases plotted in Figure 1. To proceed, we need more information on shock and rarefaction discontinuities. Shocks will be formed in the following cases:

- (i) if there is an initial negative jump;

- (ii) if $\dot{a}\left(u_{0}\right)$ has a negative minimum in an interval where $u_{0}$ is $C^{2}$ smooth;

- (iii) if $u_{0}$ has positive jump at a point, $x=z$ say, and $\dot{a}\left(u_{0}(z+)\right)$ or $\dot{a}\left(u_{0}(z-)\right)$ is a negative minimum.

A shock may also occur as a consequence of an interaction of two waves. If $x=X_{m}(t)$ and $x=X_{m+1}(t)$ are interacted with each other, then we denote the resulting shock still as $x=X_{m}(t)$. It is regarded as an extension of $x=X_{m}(t)$ or $x=X_{m+1}(t)$, rather than a new shock.

Consider first two cases. Assume $x=X(t)$ is a shock curve. Before $x=X(t)$ is interacted by other waves, say $t_{p}<t<t^{(p, n)}$, we can trace two characteristic lines 

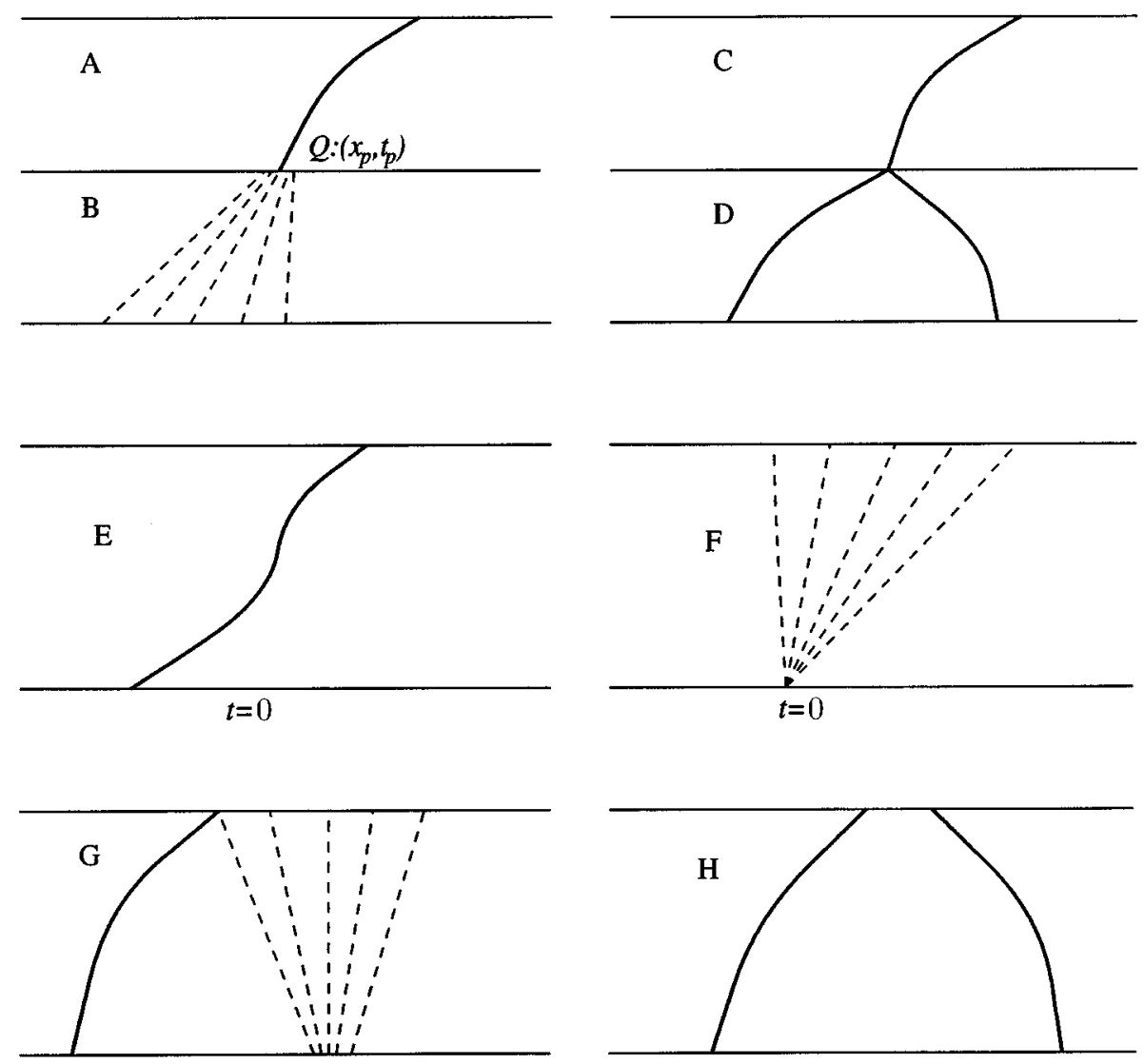

FiguRE 1. Solution structures

from $x=X(t)$ backward to $t=0$ :

$$
X(t)=\zeta^{-}+a\left(u_{0}\left(\zeta^{-}\right)\right) t=\zeta^{+}+a\left(u_{0}\left(\zeta^{+}\right)\right) t,
$$

where $\zeta^{+}(t)>\zeta^{-}(t)$. Differentiating both sides of the first identity in (1.1) with respect to $t$ gives

$$
\dot{\zeta}^{-}=\frac{\dot{X}(t)-a\left(u_{0}\left(\zeta^{-}\right)\right)}{1+\dot{a}\left(u_{0}\left(\zeta^{-}\right)\right) t}, \quad \dot{\zeta}^{-}:=\frac{d \zeta^{-}(t)}{d t}
$$

or equivalently

$$
\frac{1}{1+\dot{a}\left(u_{0}\left(\zeta^{-}\right)\right) t}=\frac{\dot{\zeta}^{-}}{\dot{X}(t)-a\left(u_{0}\left(\zeta^{-}\right)\right)} .
$$

Observe that

$$
\begin{aligned}
\dot{X}(t)-a\left(u_{0}\left(\zeta^{-}\right)\right) & =\int_{0}^{1} a\left(\theta u^{+}+(1-\theta) u^{-}\right) d \theta-a\left(u^{-}\right) \\
& =\int_{0}^{1} f^{\prime \prime}(\xi) \theta d \theta\left(u^{+}-u^{-}\right),
\end{aligned}
$$


where $u^{ \pm}=u(X(t) \pm 0, t), \xi$ denotes some intermediate value. Therefore, we have

$$
\left|\dot{X}(t)-a\left(u_{0}\left(\zeta^{-}\right)\right)\right| \geq c\left|u^{+}-u^{-}\right| \geq c\left|a\left(u^{+}\right)-a\left(u^{-}\right)\right|=\frac{c}{t}\left|\zeta^{+}-\zeta^{-}\right|,
$$

where $c$ is some constant independent of $t$, and in the last step we have used (1.1). The above results lead to

$$
\frac{1}{1+\dot{a}\left(u_{0}\left(\zeta^{-}\right)\right) t} \leq \frac{c t\left|\dot{\zeta}^{-}\right|}{\mid \zeta^{+}-\zeta^{-1}}
$$

Graphically, it is easy to see that $\zeta^{-}$is a decreasing function of $t$ and $\zeta^{+}$is an increasing function of $t$. Therefore, we have $\left|\dot{\zeta}^{-}\right|=-\dot{\zeta}^{-} \leq \dot{\zeta}^{+}-\dot{\zeta}^{-}$. It is also true that $\zeta^{+}>\zeta^{-}$. Therefore, we have

$$
\frac{1}{1+\dot{a}\left(u_{0}\left(\zeta^{-}\right)\right) t} \leq \frac{c t\left(\dot{\zeta}^{+}-\dot{\zeta}^{-}\right)}{\zeta^{+}-\zeta^{-}} .
$$

A similar result holds for $\zeta^{+}$. Consequently, we conclude for both Cases (i) and (ii) that

$$
\frac{1}{1+\dot{a}\left(u_{0}\left(\zeta^{ \pm}\right)\right) t} \leq \frac{c t\left(\dot{\zeta}^{+}-\dot{\zeta}^{-}\right)}{\zeta^{+}-\zeta^{-}}
$$

where $c$ is a constant independent of $t$, and the inequality holds before $x=X(t)$ collides with another shock.

Remark 1.3. The inequality (1.5) is an important result. Assume that $x=X(t)$ is not interacted by other waves for $t \in(S, T)$. It follows from (1.5) that

$$
\begin{aligned}
& \int_{S}^{T} \frac{1}{1+\dot{a}\left(u_{0}\left(\zeta^{ \pm}\right)\right) t} d t \\
\leq & C(T)\left(\left|\log \left(\zeta^{+}(T)-\zeta^{-}(T)\right)\right|+\left|\log \left(\zeta^{+}(S)-\zeta^{-}(S)\right)\right|\right) .
\end{aligned}
$$

For a finite $T$, we have

$$
\zeta^{+}(S)-\zeta^{-}(S) \leq \zeta^{+}(T)-\zeta^{-}(T) \leq C .
$$

To bound the right-hand side of (1.6), we only need to find the lower bound for $\zeta^{+}(S)-\zeta^{-}(S)$. See, for example, step 1 in the proof of Lemma 1.1.

In Case (iii), a new shock is generated on the boundary of a central rarefaction wave. Since one side of the resulting shock is within the rarefaction wave, one of the two characteristic lines from any point on the shock will end up at the positive jump point. Consider, for example, $u_{0}$ has positive jump at $x=z$ and $\dot{a}\left(u_{0}(z-)\right)$ is a negative minimum in the sense that $\dot{a}\left(u_{0}(z-)\right)<\dot{a}\left(u_{0}(x)\right)$ for $x$ in the left neighborhood of $z$. There we will have a central rarefaction wave with boundaries $x=X_{L}(t)$ and $x=X_{R}(t)$. Starting at the point $\left(X_{L}\left(t_{z}\right), t_{z}\right)$, where $t_{z}=-1 / \dot{a}\left(u_{0}(z-)\right)$, a new shock $x=X(t)$ will be generated. It satisfies

$$
X(t)=\zeta^{-}+a\left(u_{0}\left(\zeta^{-}\right)\right) t .
$$

Now (1.3) holds, with

$$
\dot{X}(t)=\int_{0}^{1} a\left(\theta u^{+}+(1-\theta) u^{-}\right) d \theta,
$$


where $u^{+}=a^{-1}((X(t)-z) / t), u^{-}=u(X(t)-0, t)$. It follows from (1.4) and (1.7) that

$$
\begin{aligned}
\left|\dot{X}(t)-a\left(u_{0}\left(\zeta^{-}\right)\right)\right| & \geq c\left|u^{+}-u^{-}\right| \geq c\left|a\left(u^{+}\right)-a\left(u^{-}\right)\right| \\
& =\frac{c}{t}\left|(X(t)-z)-a\left(u_{0}\left(\zeta^{-}\right)\right) t\right|=\frac{c}{t}\left|\zeta^{-}-z\right| .
\end{aligned}
$$

Therefore, we have

$$
\frac{1}{1+\dot{a}\left(u_{0}\left(\zeta^{-}\right)\right) t} \leq \frac{c t \dot{\zeta}^{-}}{\zeta^{-}-z}
$$

Similarly, if $u_{0}$ has positive jump at $x=z$ and $\dot{a}\left(u_{0}(z+)\right)$ is a negative minimum, we have

$$
\frac{1}{1+\dot{a}\left(u_{0}\left(\zeta^{+}\right)\right) t} \leq \frac{c t \dot{\zeta}^{+}}{\zeta^{+}-z}
$$

Remark 1.4. Similar to Remark 1.3, it follows from (1.8) that

$$
\begin{aligned}
& \int_{S}^{T} \frac{1}{1+\dot{a}\left(u_{0}\left(\zeta^{-}\right)\right) t} d t \\
\leq & C(T)\left(\left|\log \left(\zeta^{-}(T)-z\right)\right|+\left|\log \left(\zeta^{-}(S)-z\right)\right|\right) .
\end{aligned}
$$

Therefore, it is important to find a bound for $\zeta^{-}(S)-z$. See, for example, step 1 in the proof of Lemma 1.1. A similar idea will be used to treat (1.9).

From the above discussion, we can obtain the following lemma which is important in the error analysis.

Lemma 1.1. Assume $x=X(t)$ is a shock curve satisfying

$$
\dot{X}(t)=\frac{f\left(u^{+}\right)-f\left(u_{-}\right)}{u^{+}-u^{-}}, \quad u^{ \pm}:=u(X(t) \pm 0, t) .
$$

Assume $a\left(u_{0}\right)$ is sufficiently smooth in the neighborhood of its negative minimum points. If $x=X(t)$ is formed at $t=0$, then

$$
\int_{0}^{T}\left|u_{x}(X(t) \pm 0, t)\right| d t \leq C .
$$

If $x=X(t)$ is formed at $t=t_{p}>0$, then

$$
\int_{t_{p}+\delta}^{T}\left|u_{x}(X(t) \pm 0, t)\right| d t \leq C|\log \delta|+C,
$$

provided that $\delta>0$ is sufficiently small. Here $T>t_{P}$ is a fixed number.

Proof. The proof of this lemma consists of three steps.

Step 1: Initial negative jump. In this case, a shock is formed due to initial negative jump at a point, say $x=b$. It is obvious that this shock is not going to be interacted by other waves before a finite time $t^{(0, n)}, 1 \leq n \leq N_{0}$. It is known that

$$
u\left(\zeta^{ \pm}+a\left(u_{0}\left(\zeta^{ \pm}\right)\right) t, t\right)=u_{0}\left(\zeta^{ \pm}\right), \quad 0<t<t^{(0, n)} .
$$

Differentiating both sides with resepect to $\zeta^{+}$or $\zeta^{-}$gives

$$
u_{x}(X(t) \pm 0, t)=\frac{\dot{u}_{0}\left(\zeta^{ \pm}\right)}{1+\dot{a}\left(u_{0}\left(\zeta^{ \pm}\right)\right) t} .
$$


Since $\dot{a}\left(u_{0}(x \pm 0)\right)$ is bounded by a constant independent of $x$, there exists a constant $\eta$ such that $\left|u_{x}(X(t) \pm 0, t)\right| \leq c$ for all $0<t<\eta$. If this shock collides with another wave at $t=t^{(0, n)}$, we choose a positive $\gamma$ satisfying $\gamma<\min \left(\eta, t^{(0, n)}\right)$. Obviously,

$$
\int_{0}^{\gamma}\left|u_{x}(X(t) \pm 0, t)\right| d t \leq C
$$

Again, since $\zeta^{-}$is decreasing and $\zeta^{+}$is increasing, we have

$$
\zeta^{+}(t)-\zeta^{-}(t) \geq \zeta^{+}(\gamma)-\zeta^{-}(\gamma)=\text { constant }>0, \quad \text { for } t \in\left(\gamma, t^{(0, n)}\right) .
$$

Using Remark 1.3 and the above inequality, we can obtain that

$$
\int_{\gamma}^{t^{(0, n)}}\left|u_{x}(X(t) \pm 0, t)\right| d t \leq C .
$$

At $t=t^{(0, n)}$, if the shock is interacted by another shock, then the resulting shock satisfies

$$
\zeta^{+}(t)-\zeta^{-}(t) \geq \zeta^{+}(\gamma)-\zeta^{-}(\gamma)=\text { constant }>0, \quad \text { for } t \in\left(t^{(0, n)}, t^{\left(0, n^{\prime}\right)}\right),
$$

where $t^{\left(0, n^{\prime}\right)} \in\left(t^{(0, n)}, t_{1}\right)$ is the next (possible) shock interaction time. Again, using Remark 1.3 and the above inequality gives

$$
\int_{t^{(0, n)}}^{t^{\left(0, n^{\prime}\right)}}\left|u_{x}(X(t) \pm 0, t)\right| d t \leq C .
$$

At $t=t^{(0, n)}$, if the shock is interacted by a central rarefaction wave, $u(x, t)=$ $a^{-1}((x-z) / t)$, at $t=t^{(0, n)}$, say on the left boundary as in Figure $1(\mathrm{G})$, then we have

$$
z-\zeta^{-}(t) \geq z-\zeta^{-}\left(t^{(0, n)}\right) \geq z-\zeta^{-}(\gamma)=\text { constant }>0, \quad \text { for } t \in\left(t^{(0, n)}, t^{\left(0, n^{\prime}\right)}\right) .
$$

Using Remark 1.4 and the fact that

$$
\left|u_{x}(X(t)+0, t)\right| \leq C / t \quad \text { for } \quad t \in\left(t^{(0, n)}, t^{\left(0, n^{\prime}\right)}\right)
$$

gives the same estimate as (1.17). Repeating this procedure a finite number of times, we can obtain (1.12).

Step 2: Negative minimum point in a smooth domain. Assume that $\zeta_{p} \in$ $\left(z_{1}, z_{2}\right)$ is a negative minimum point of $\dot{a}\left(u_{0}\right)$, and $a\left(u_{0}\right)$ is sufficiently smooth in $\left(z_{1}, z_{2}\right)$. Then there is a shock curve, $x=X(t)$, formed at $\left(x_{p}, t_{p}\right)$, where $x_{p}=\zeta_{p}+a\left(u_{0}\left(\zeta_{p}\right)\right) t_{p}$ with $t_{p}=-1 / \dot{a}\left(u_{0}\left(\zeta_{p}\right)\right)$. From each point away from the curve, we can trace a characteristic line backward in time to $t=0$. Using Lax' geometric condition, we know that these lines will not end up with the discontinuous points. Let $x=\zeta+a\left(u_{0}(\zeta)\right) t$ denote a characteristic line passing through an $(x, t)$ point. We point out that $\zeta$ is a function of $x$ and $t$. For a fixed time $t$, it is easy to see that a larger value of $x$ corresponds to a larger value of $\zeta$. This gives

$$
\frac{\partial x}{\partial \zeta}=1+\dot{a}\left(u_{0}(\zeta)\right) t \geq 0, \quad \dot{a}\left(u_{0}(\zeta)\right):=\frac{d}{d \zeta}\left(a\left(u_{0}(\zeta)\right)\right)
$$

See also Dafermos [2] for a similar result. Notice that $\zeta_{p}$ is a negative minimum point of $\dot{a}\left(u_{0}(\zeta)\right)$. This suggests that there exists a positive integer $s$ such that $a^{(k)}\left(u_{0}\left(\zeta_{p}\right)\right)=0,2 \leq k \leq 2 s$, and $a^{(2 s+1)}\left(u_{0}\left(\zeta_{p}\right)\right)>0$, where

$$
a^{(k)}\left(u_{0}(x)\right):=\frac{d^{k}}{d x^{k}} a\left(u_{0}(x)\right) .
$$


Moreover, there exists a constant $\gamma>0$ such that

$$
0<a^{(2 s+1)}\left(u_{0}(\zeta)\right) \leq 2 a^{(2 s+1)}\left(u_{0}\left(\zeta_{p}\right)\right), \quad \text { for }\left|\zeta-\zeta_{p}\right|<\gamma .
$$

From (1.18) and Taylor's expansion, we obtain

$$
1+\dot{a}\left(u_{0}\left(\zeta_{p}\right)\right) t+\frac{1}{(2 s) !} a^{(2 s+1)}\left(u_{0}(\xi)\right)\left(\zeta-\zeta_{p}\right)^{2 s} t \geq 0,
$$

where $\xi$ is between $\zeta$ and $\zeta_{p}$. Using the fact that $\dot{a}\left(u_{0}\left(\zeta_{p}\right)\right)=-1 / t_{p}$, we obtain that

$$
1-\frac{t}{t_{p}}+\frac{1}{(2 s) !} a^{(2 s+1)}\left(u_{0}(\xi)\right)\left(\zeta-\zeta_{p}\right)^{2 s} t \geq 0 .
$$

If $\left|\zeta-\zeta_{p}\right|<\gamma$, we obtain from (1.19) and (1.20) that

$$
\left|\zeta-\zeta_{p}\right| \geq C\left(t-t_{p}\right)^{\frac{1}{2 s}}, \quad t_{p}<t<t^{(p, n)},
$$

where $t^{(p, n)} \leq t_{p+1}$ is the smallest time level when $x=X(t)$ collides with other waves $\left(t^{(p, n)}=t_{p+1}\right.$ if this does not happen). Therefore, we have proved that for all $\zeta$ near $\zeta_{p}$,

$$
\left|\zeta-\zeta_{p}\right| \geq \min \left\{\gamma, C\left(t-t_{p}\right)^{\frac{1}{2 s}}\right\}, \quad t_{p}<t<t^{(p, n)} .
$$

Tracing two characteristic lines from $x=X(t)$ backward to $t=0$, see (1.1), we obtain from (1.21)

$$
\zeta^{+}(t)-\zeta^{-}(t) \geq \min \left\{\gamma, C\left(t-t_{p}\right)^{\frac{1}{2 s}}\right\}, \quad t_{p}<t<t^{(p, n)} .
$$

From (1.14), (1.22) and Remark 1.3, we obtain

$$
\int_{\delta+t_{p}}^{t^{(p, n)}}\left|u_{x}(X(t) \pm 0, t)\right| d t \leq C|\log \delta|+C,
$$

where $\delta>0$ is sufficiently small. By using the same argument as used in step 1 of this proof, we can extend the above integration to the time interval $\left(t_{p}+\delta, T\right)$.

Step 3: Negative minimum point at a positive jump endpoint. Without loss of generality, we assume that $u_{0}$ has a positive jump at $x=z$, and $\dot{a}\left(u_{0}(z-)\right)$ is a negative minimum. Let $X_{L}(t)$ be the left boundary of the central rarefaction wave and $t_{p}=-1 / \dot{a}\left(u_{0}(z-)\right)$. Then a shock $x=X(t)$ is formed at the point $\left(x_{p}, t_{p}\right)$, where $x_{p}=X_{L}\left(t_{p}\right)$. Assuming that $a\left(u_{0}(\zeta)\right)$ is sufficiently smooth as $\zeta \rightarrow z-0$ and using Taylor's expansion, we have

$$
z-\zeta^{-}(t) \geq \min \left\{\gamma, C\left(t-t_{p}\right)^{\frac{1}{2 s}}\right\}
$$

where $s$ is a positive constant. This, together with Remark 1.4, yield that

$$
\int_{\delta+t_{p}}^{t^{(0, n)}}\left|u_{x}(X(t)-0, t)\right| d t \leq C|\log \delta|+C
$$

where $t^{(0, n)}$ indicates the smallest time level when the shock collides with another wave. It is clear that $u(x, t)=\left(f^{\prime}\right)^{-1}((x-z) / t)$ for $x \rightarrow X(t)+0$ and hence $\left|u_{x}(X(t)+0, t)\right| \leq C / t$. We can then conclude that (1.13) is true for this case. 
Remark 1.5. It is observed from the proof that if the discontinuities are purely due to the initial negative jump, then the $O(|\log \delta|)$ factor is not included in the estimate for the integral of $u_{x}$, see (1.12). The optimal error estimate seems possible in the case when the solution $u$ contains neither a central rarefaction wave nor an original shock generated at a later time. This will be investigated in Section 4 .

Remark 1.6. Assume $x=X(t)$ is a shock curve generated at $t=0$ or $t=t_{p}$ and $x=\zeta^{ \pm}+a\left(u_{0}\left(\zeta^{ \pm}\right)\right) t$ are the corresponding characteristic lines. We have proved in the above lemma that

$$
\int_{t_{p}+\delta}^{T} \frac{1}{1+\dot{a}\left(u_{0}\left(\zeta^{ \pm}\right)\right) t} d t \leq C|\log \delta|+C .
$$

The partial derivative $u_{x}$ will also be discontinuous at a point $z$ if there is an initial positive jump at $z$, i.e. $u_{0}(z+0)>u_{0}(z-0)$. This corresponds to a central rarefaction wave. The case that the rarefaction wave collides with a shock is included in Step 1 of the proof for Lemma 1.1. Here we are interested in the central rarefaction wave before it is interacted by a shock.

Lemma 1.2. Assume a central rarefaction wave is formed at $x=z$. Let $x=X_{L}(t)$ and $x=X_{R}(t)$ be left and right boundaries of the rarefaction wave, respectively. If $\dot{a}\left(u_{0}(z+0)\right)$ is not a negative minimum, then

$$
\left|u_{x}\left(X_{R}(t)+0, t\right)\right| \leq C, \quad\left|u_{x}\left(X_{R}(t)-0, t\right)\right| \leq C t^{-1} .
$$

The above results hold before the rarefaction wave is interacted by a shock. If $\dot{a}\left(u_{0}(z+0)\right)$ is a negative minimum, then

$$
\left|u_{x}\left(X_{R}(t)+0, t\right)\right| \leq C\left(t-t_{p}\right)^{-1}, \quad\left|u_{x}\left(X_{R}(t)-0, t\right)\right| \leq C t^{-1},
$$

for $t<t_{p}$, where $t_{p}=-1 / \dot{a}\left(u_{0}(z+0)\right)$. The curve $x=X_{R}(t)$ will become a shock after $t=t_{p}$. Similar results, based on $\dot{a}\left(u_{0}(z-0)\right)$, hold for $X_{L}(t)$.

Proof. Observe that

$$
u(x, t)=\left(f^{\prime}\right)^{-1}\left(\frac{x-z}{t}\right), \quad X_{L}(t) \leq x \leq X_{R}(t),
$$

where $X_{L}(t)=z+f^{\prime}\left(u_{0}(z-0)\right) t, X_{R}(t)=z+f^{\prime}\left(u_{0}(z+0)\right) t$. The above result gives that

$$
\left|u_{x}\left(X_{R}(t)-0, t\right)\right| \leq C t^{-1} .
$$

Moreover, if $\dot{a}\left(u_{0}(z+0)\right) \geq 0$ or $\dot{a}\left(u_{0}(z+0)\right)<0$ but not a local minimum, then we can show that $\left|u_{x}\left(X_{R}(t)+0, t\right)\right| \leq C$ before $x=X_{R}(t)$ collides with other waves. Now consider the case that $\dot{a}\left(u_{0}(z+0)\right)<0$ is a local minimum. Then a new shock will be formed at $t_{p}=-1 / \dot{a}\left(u_{0}(z+0)\right)$. Similar to (1.14) we have

$$
u_{x}\left(X_{R}(t)+0, t\right)=\frac{\dot{u}_{0}(z+0)}{1+\dot{a}\left(u_{0}(z+0)\right) t}, \quad 0<t<t_{p}
$$

This and the fact that $t_{p}=-1 / \dot{a}\left(u_{0}(z+0)\right)$ yield

$$
\left|u_{x}\left(X_{R}(t)+0, t\right)\right| \leq C\left(t_{p}-t\right)^{-1}, \quad 0<t<t_{p} .
$$

The proof is complete. 
Remark 1.7. Let $x=X_{R}(t)$ be the right boundary of a central rarefaction wave. It may collide with a shock at a certain time, say $t=t^{*}$. We still denote the resulting shock as $X_{R}(t)$. Hence, we have extended the curve $x=X_{R}(t)$ to the whole time interval $[0, T]$. Using Lemmas 1.1 and 1.2 gives

$$
\int_{t_{p}+\delta}^{t_{p+1}-\delta}\left|u_{x}\left(X_{R}(t) \pm 0, t\right)\right| d t \leq C|\log \delta|+C .
$$

Moreover, from each point on $x=X_{R}(t)$ we can trace a right characteristic line $x=\zeta^{+}+a\left(u_{0}\left(\zeta^{+}\right)\right) t$ back to $t=0$. Here $\zeta^{+}=z+0$ before $X_{R}(t)$ is interacted by a shock. From the proofs for Lemmas 1.1 and 1.2, we can show that

$$
\int_{t_{p}+\delta}^{t_{p+1}-\delta} \frac{1}{1+\dot{a}\left(u_{0}\left(\zeta^{+}\right)\right) t} d t \leq C|\log \delta|+C
$$

We may extend $x=X_{L}(t)$ in a similar way and trace a left characteristic line $x=\zeta^{-}+a\left(u_{0}\left(\zeta^{-}\right)\right) t$ back to $t=0$. Here, $\zeta^{-}=z-0$ before $x=X_{L}(t)$ collides with a shock. We also have

$$
\begin{gathered}
\int_{t_{p}+\delta}^{t_{p+1}-\delta}\left|u_{x}\left(X_{L}(t) \pm 0, t\right)\right| d t \leq C|\log \delta|+C, \\
\int_{t_{p}+\delta}^{t_{p+1}-\delta} \frac{1}{1+\dot{a}\left(u_{0}\left(\zeta^{-}\right)\right) t} d t \leq C|\log \delta|+C .
\end{gathered}
$$

In order to provide a sharp $L^{1}$-error bound, some properties on second-order derivatives are also needed, e.g. see Sections 3.2 and 3.3.

Lemma 1.3. Assume that $u_{0}$ satisfies the assumptions $(\mathrm{A} 1)-(\mathrm{A} 4)$ and let $a\left(u_{0}\right)$ be sufficiently smooth near its negative minimum points. Then the solution to (0.1) and (0.2) satisfies

$$
\int_{t_{p}+\delta}^{t_{p+1}-\delta}\left\|u_{x x}(\bullet, t)\right\| d t \leq C|\log \delta|+C, \quad 0 \leq p \leq P
$$

provided $\delta$ is sufficiently small.

Proof. For any fixed time $t \in\left(t_{p}, t_{p+1}\right)$, there are finite intervals $\left(Z_{l}(t), Z_{l+1}(t)\right)$ (the number of the intervals can be bounded by a constant independent of $t$ ) such that the transformation $x=\zeta+a\left(u_{0}(\zeta)\right) t$ maps $\left(Z_{l}(t), Z_{l+1}(t)\right)$ (in $x$ space) to $\left(\theta_{l}(t), \theta_{l+1}(t)\right)$ (in $\zeta$ space). The solution $u(x, t)$ is continuously differentiable in $\left(Z_{l}, Z_{l+1}\right)$ but not at the end points $Z_{l}(t)$ and $Z_{l+1}(t)$. It is observed that

$$
\min _{l} Z_{l}(t)=\min _{l} \theta_{l}=-\infty, \quad \text { and } \max _{l} Z_{l}(t)=\max _{l} \theta_{l}=\infty .
$$

In the case that $Z_{l}(t)$ and $Z_{l+1}(t)$ are boundaries of a central rarefaction wave, there exists an integer $s$ such that $\theta_{s}(t)=\theta_{s+1}(t)=z_{s}$ and the solution is of the form

$$
u(x, t)=\left(f^{\prime}\right)^{-1}\left(\frac{x-z_{s}}{t}\right), \quad Z_{l}(t) \leq x \leq Z_{l+1}(t),
$$

where $Z_{l+1}(t)-Z_{l}(t) \leq\left[f^{\prime}\left(u_{0}\left(z_{s}+\right)\right)-f^{\prime}\left(u_{0}\left(z_{s}-\right)\right)\right] t$. It follows from $f^{\prime \prime} \geq \alpha>0$ that $\left[\left(f^{\prime}\right)^{-1}\right]^{\prime \prime}(\zeta) \leq C$ for $|\zeta| \leq C$. Hence, we have

$$
\int_{Z_{l}(t)}^{Z_{l+1}(t)}\left|u_{x x}(x, t)\right| d x \leq C \int_{Z_{l}(t)}^{Z_{l+1}(t)} \frac{d x}{t^{2}}=C\left(Z_{l+1}(t)-Z_{l}(t)\right) t^{-2} \leq C t^{-1},
$$


which leads to

$$
\int_{t_{p}+\delta}^{t_{p+1}} \int_{Z_{l}(t)}^{Z_{l+1}(t)}\left|u_{x x}(x, t)\right| d x d t \leq C|\log \delta|+C .
$$

If $\theta_{l}<\theta_{l+1}$, we have $1+\dot{a}\left(u_{0}(\zeta)\right) t>0$ for $\zeta \in\left(\theta_{l}, \theta_{l+1}\right)$. Differentiating the equation $u\left(\zeta+a\left(u_{0}(\zeta)\right) t, t\right)=u_{0}(\zeta)$ with respect to $\zeta$ gives

$$
u_{x x}\left(\zeta+a\left(u_{0}(\zeta)\right) t, t\right)\left(1+\dot{a}\left(u_{0}\right) t\right)=-\frac{\dot{u_{0}} \ddot{a}\left(u_{0}\right) t}{\left(1+\dot{a}\left(u_{0}\right) t\right)^{2}}+\frac{\ddot{u}_{0}}{1+\dot{a}\left(u_{0}\right) t} .
$$

It follows from the above result that

$$
\begin{aligned}
\int_{Z_{l}(t)}^{Z_{l+1}(t)}\left|u_{x x}(x, t)\right| d x & =\int_{\theta_{l}(t)}^{\theta_{l+1}(t)}\left|u_{x x}\left(\zeta+a\left(u_{0}(\zeta)\right) t, t\right)\left(1+\dot{a}\left(u_{0}\right) t\right)\right| d \zeta \\
& \leq \int_{\theta_{l}(t)}^{\theta_{l+1}(t)} \frac{\left|\dot{u} 0 \ddot{a}\left(u_{0}\right)\right| t}{\left(1+\dot{a}\left(u_{0}\right) t\right)^{2}} d \zeta+\int_{\theta_{l}(t)}^{\theta_{l+1}(t)} \frac{\left|\ddot{u}_{0}\right|}{1+\dot{a}\left(u_{0}\right) t} d \zeta \\
& =: I_{1}+I_{2} .
\end{aligned}
$$

Observe that

$$
\frac{\partial}{\partial \zeta}\left(\frac{1}{1+\dot{a}\left(u_{0}\right) t}\right)=-\frac{\ddot{a}\left(u_{0}\right) t}{\left(1+\dot{a}\left(u_{0}\right) t\right)^{2}} .
$$

This suggests that the maximum of $1 /\left(1+\dot{a}\left(u_{0}\right) t\right)$ possibly occurs at (i) increasing inflection points of $a\left(u_{0}\right)$; (ii) decreasing inflection points of $a\left(u_{0}\right)$; and (iii) the end points $\theta_{l}(t)$ or $\theta_{l+1}(t)$. For the possibility (i), we have $1 /\left(1+\dot{a}\left(u_{0}\right) t\right) \leq 1$. For the possibility (ii), noting that $1+\dot{a}\left(u_{0}\right) t>0$, we have

$$
\frac{1}{1+\dot{a}\left(u_{0}\right) t} \leq \max _{t_{j}>t} \frac{1}{1-t / t_{j}}
$$

Since $t \in\left(t_{p}, t_{p+1}\right)$, we obtain from the above result that

$$
\frac{1}{1+\dot{a}\left(u_{0}\right) t} \leq \frac{C}{t_{p+1}-t} .
$$

Consequently, we obtain for the above three possibilities that

$$
\frac{1}{1+\dot{a}\left(u_{0}\right) t} \leq 1+\frac{C}{t_{p+1}-t}+\frac{1}{1+\dot{a}\left(u_{0}\left(\theta_{l}\right)\right) t}+\frac{1}{1+\dot{a}\left(u_{0}\left(\theta_{l+1}\right)\right) t} .
$$

Notice that there is no new shock being generated for $t \in\left(t_{p}, t_{p+1}\right)$. If $x=Z_{l}(t)$ corresponds to a shock curve, then $\theta_{l}(t)=\zeta^{+}(t)$ following our notation in Lemma 1.1. Then using Remark 1.6 we have

$$
\int_{t_{p}+\delta}^{t_{p+1}} \frac{1}{1+\dot{a}\left(u_{0}\left(\theta_{l}\right)\right) t} d t \leq C|\log \delta|+C
$$

If $x=Z_{l}(t)$ is a right boundary of a rarefaction wave and collides with a shock at $t=t^{*} \in\left(t_{p}, t_{p+1}\right)$, then using Remark 1.7 gives

$$
\int_{t_{p}+\delta}^{t_{p+1}-\delta} \frac{1}{1+\dot{a}\left(u_{0}\left(\theta_{l}\right)\right) t} d t \leq C|\log \delta|+C
$$

Similar results hold for the last term in (1.29). These, together with the assumption (A2), yield

$$
\int_{t_{p}+\delta}^{t_{p+1}-\delta}\left|I_{2}\right| d t \leq C|\log \delta|+C
$$


Observe that

$$
\int_{c}^{b} \frac{\ddot{a}\left(u_{0}\right) t}{\left(1+\dot{a}\left(u_{0}\right) t\right)^{2}} d \zeta=\frac{1}{1+\dot{a}\left(u_{0}(c)\right) t}-\frac{1}{1+\dot{a}\left(u_{0}(b)\right) t}
$$

provided that $a\left(u_{0}\right) \in C^{2}(b, c)$. It follows from (A3), (A4) and the above identity that

$$
\begin{aligned}
& \int_{\theta_{l}(t)}^{\theta_{l+1}(t)} \frac{\left|\ddot{a}\left(u_{0}\right)\right| t}{\left(1+\dot{a}\left(u_{0}\right) t\right)^{2}} d \zeta \\
\leq & \frac{1}{1+\dot{a}\left(u_{0}\left(\theta_{l}(t)\right)\right) t}+\frac{1}{1+\dot{a}\left(u_{0}\left(\theta_{l+1}(t)\right)\right) t}+\sum_{j} \frac{2}{1+\dot{a}\left(u_{0}\left(y_{j}\right)\right) t},
\end{aligned}
$$

where $y_{j} \in\left(\theta_{j}, \theta_{j+1}\right)$ are inflection points of $a\left(u_{0}\right)$. Similar to the discussion for $I_{2}$, we have

$$
\int_{t_{p}+\delta}^{t_{p+1}-\delta} \int_{\theta_{l}(t)}^{\theta_{l+1}(t)} \frac{\left|\ddot{a}\left(u_{0}\right)\right| t}{\left(1+\dot{a}\left(u_{0}\right) t\right)^{2}} d \zeta d t \leq C|\log \delta|+C .
$$

Using (A1) and (A2), we can show that $\dot{u}_{0}$ is bounded almost everywhere. This and the above inequality yield that

$$
\int_{t_{p}+\delta}^{t_{p+1}-\delta}\left|I_{1}\right| d t \leq C|\log \delta|+C
$$

The proof of Lemma 1.3 is thereby complete.

\section{Stability AND TRAVELING WAVE}

Our error estimates are based on a stability lemma for nonhomogeneous viscous equations and a traveling wave lemma. We first introduce the stability lemma.

Lemma 2.1. Let $v^{(i)}(x, t), i=1,2$, be continuous and piecewise smooth solutions of the following equations:

$$
\left(v^{(i)}\right)_{t}+\left(f\left(v^{(i)}\right)\right)_{x}-\epsilon\left(v^{(i)}\right)_{x x}=g_{i}(x, t), \quad t>a, \quad i=1,2 .
$$

The above equation holds for all values of $x$ except on some curves $X_{m}(t), 1 \leq m \leq$ $M$, where $v_{x}^{(i)}$ may not exist. If $w:=v^{(1)}-v^{(2)} \rightarrow 0$ as $x \rightarrow \infty$, then

$$
\begin{aligned}
\|w(\bullet, t)\| \leq\|w(\bullet, a)\| & +\int_{a}^{t}\left\|g_{1}(\bullet, \tau)-g_{2}(\bullet, \tau)\right\| d \tau \\
& +\epsilon \sum_{m=1}^{M} \int_{a}^{t}\left|w_{x}\left(X_{m}(\tau)+0, \tau\right)-w_{x}\left(X_{m}(\tau)-0, \tau\right)\right| d \tau
\end{aligned}
$$

Proof. It follows from (2.1) that

$$
w_{t}+\left(f^{\prime}(\zeta) w\right)_{x}-\epsilon w_{x x}=g_{1}(x, t)-g_{2}(x, t),
$$

where $\zeta$ is some intermediate value between $v^{(1)}$ and $v^{(2)}$. If $w \geq 0$ or $w \leq 0$ for all $x$, then straightforward integration on the above equation gives (2.2). Let $p_{0}(t)<p_{1}(t)<p_{2}(t)<\cdots$ be the points such that at those points $w$ changes signs. 
Let $\alpha_{j}$ be the sign of $w$ in $\left(p_{j}, p_{j+1}\right)$. Multiplying (2.3) by $\alpha_{j}$ and integrating the resulting equation over $\left(p_{j}, p_{j+1}\right)$ gives

$$
\begin{aligned}
& \alpha_{j} \int_{p_{j}}^{p_{j+1}} w_{t} d x=\epsilon\left(\alpha_{j} w_{x}\left(p_{j+1}-0, t\right)-\alpha_{j} w_{x}\left(p_{j}+0, t\right)\right) \\
& \quad+\sum_{p_{j}<X_{m}<p_{j+1}} \epsilon \alpha_{j}\left(w_{x}\left(X_{m}(t)+0, t\right)-w_{x}\left(X_{m}-0, t\right)\right) \\
& \quad+\alpha_{j} \int_{p_{j}}^{p_{j+1}}\left(g_{1}(x, t)-g_{2}(x, t)\right) d x .
\end{aligned}
$$

Since $w\left(p_{j}, t\right)=w\left(p_{j+1}, t\right)=0$ and $\alpha_{j} w \geq 0$ for $x \in\left(p_{j}, p_{j+1}\right)$, we have

$$
\frac{d}{d t} \int_{p_{j}}^{p_{j+1}}|w| d x=\alpha_{j} \int_{p_{j}}^{p_{j+1}} w_{t} d x
$$

Moreover, observing that $\alpha_{j} w_{x}\left(p_{j+1}-0, t\right) \leq 0$ and $\alpha_{j} w_{x}\left(p_{j}+0, t\right) \geq 0$, we obtain from (2.4) that

$$
\begin{aligned}
\frac{d}{d t} \int_{p_{j}}^{p_{j+1}}|w| d x \leq & \sum_{p_{j}<X_{m}<p_{j+1}} \epsilon\left|w_{x}\left(X_{m}+0, t\right)-w_{x}\left(X_{m}-0, t\right)\right| \\
& +\int_{p_{j}}^{p_{j+1}}\left|g_{1}(x, t)-g_{2}(x, t)\right| d x .
\end{aligned}
$$

Since the above inequality is true for all $j \geq 0$, we have

$$
\begin{aligned}
\frac{d}{d t} \int_{p_{0}}^{p^{*}}|w| d x \leq & \sum_{p_{0}<X_{m}<p^{*}} \epsilon\left|w_{x}\left(X_{m}+0, t\right)-w_{x}\left(X_{m}-0, t\right)\right| \\
& +\int_{p_{0}}^{p^{*}}\left|g_{1}(x, t)-g_{2}(x, t)\right| d x,
\end{aligned}
$$

where $p^{*}=\sup _{j} p_{j}$. If $p^{*}<\infty$, using a similar method as above gives

$$
\begin{gathered}
\frac{d}{d t} \int_{p^{*}}^{\infty}|w| d x \leq \epsilon \sum_{X_{m}>p^{*}}\left|w_{x}\left(X_{m}(t)+0, t\right)-w_{x}\left(X_{m}-0, t\right)\right| \\
+\int_{p^{*}}^{\infty}\left|g_{1}(x, t)-g_{2}(x, t)\right| d x
\end{gathered}
$$

In obtaning the last inequality, we have used the fact that $w \rightarrow 0$ as $x \rightarrow \infty$. Using the above two inequalities, we obtain

$$
\begin{aligned}
\int_{p_{0}}^{\infty}|w(x, t)| d x \leq \epsilon & \sum_{X_{m}>p_{0}} \int_{a}^{t}\left|w_{x}\left(X_{m}+0, \tau\right)-w_{x}\left(X_{m}-0, \tau\right)\right| d \tau \\
& +\int_{a}^{t} \int_{p_{0}}^{\infty}\left|g_{1}(x, \tau)-g_{2}(x, \tau)\right| d x d \tau+\int_{p_{0}}^{\infty}|w(x, a)| d x .
\end{aligned}
$$

A similar result which replaces $p_{0}, \infty$ by $-\infty, p_{0}$, respectively, in the above inequality, can be established. These results lead to (2.2).

Assume $X_{m}(t)$ is a smooth curve satisfying the Rankine-Hugoniot jump condition:

$$
\dot{X}_{m}(t)=\frac{f\left(u_{m}^{+}\right)-f\left(u_{m}^{-}\right)}{u_{m}^{+}-u_{m}^{-}},
$$


where $\dot{X}(t):=d X / d t$ is the shock speed, $u_{m}^{+}=u\left(X_{m}(t)+, 0\right), u_{m}^{-}=u\left(X_{m}(t)-, 0\right)$ with $u_{m}^{+}<u_{m}^{-}$. If $u_{m}^{ \pm}$are independent of $t$, it is shown in [20] that there is a traveling wave solution of the form $V_{\epsilon}(x-S t)$, where the wave speed $S$ is a constant, satisfying $(0.3)$ and $V_{\epsilon}(-\infty)=u_{m}^{-}, V_{\epsilon}(\infty)=u_{m}^{+}$. In the case when $u_{m}^{ \pm}$are functions of $t$, we need the following generalization.

Lemma 2.2. Let $u_{m}^{+}(t)<u_{m}^{-}(t)$ be two given functions and $X_{m}(t)$ be defined by (2.5). Let $V\left(\zeta ; u_{m}^{+}, u_{m}^{-}\right)$be defined implicitly by

$$
\begin{aligned}
& \zeta=\int_{\frac{1}{2}\left(u_{m}^{+}+u_{m}^{-}\right)}^{V}\left[\Phi\left(u ; u_{m}^{+}, u_{m}^{-}\right)\right]^{-1} d u, \\
& \Phi\left(u ; u_{m}^{+}, u_{m}^{-}\right)=f(u)-f\left(u_{m}^{-}\right)-\dot{X}_{m}\left(u-u_{m}^{-}\right) .
\end{aligned}
$$

With respect to $\zeta$, the function $V\left(\zeta ; u_{m}^{+}, u_{m}^{-}\right)$is a decreasing function satisfying

$$
V\left(-\infty ; u_{m}^{+}, u_{m}^{-}\right)=u_{m}^{-}, \quad V\left(0 ; u_{m}^{+}, u_{m}^{-}\right)=\frac{1}{2}\left(u_{m}^{+}+u_{m}^{-}\right), \quad V\left(\infty ; u_{m}^{+}, u_{m}^{-}\right)=u_{m}^{+} .
$$

It also satisfies the following inequalities

$$
\begin{aligned}
& \left|V\left(\zeta ; u_{m}^{+}, u_{m}^{-}\right)-H\left(\zeta ; u_{m}^{+},, u_{m}^{-}\right)\right| \leq\left(u_{m}^{-}-u_{m}^{+}\right) \exp \left\{-\alpha\left(u_{m}^{-}-u_{m}^{+}\right)|\zeta| / 2\right\} \\
& \left\|V^{(\epsilon)}\left(\bullet-X_{m} ; u_{m}^{+}, u_{m}^{-}\right)-H\left(\bullet-X_{m} ; u_{m}^{+}, u_{m}^{-}\right)\right\| \leq \frac{4}{\alpha} \epsilon
\end{aligned}
$$

where $\alpha$ is defined by (0.5). In the above inequalities, $V^{(\epsilon)}\left(\zeta ; u_{m}^{+}, u_{m}^{-}\right):=$ $V\left(\zeta / \epsilon ; u_{m}^{+}, u_{m}^{-}\right), H$ is the so-called Heaviside function defined by

$$
H\left(x ; u_{m}^{+}, u_{m}^{-}\right)=\left\{\begin{array}{l}
u_{m}^{+} \quad \text { if } \quad x \geq 0 \\
u_{m}^{-} \quad \text { if } \quad x<0 .
\end{array}\right.
$$

Denote $V^{(\epsilon)}\left(x-X_{m}(t) ; u_{m}^{+}, u_{m}^{-}\right)$by $V_{m}^{(\epsilon)}$. Direct calculation shows that

$$
\left(V_{m}^{(\epsilon)}\right)_{t}+f\left(V_{m}^{(\epsilon)}\right)_{x}-\epsilon\left(V_{m}^{(\epsilon)}\right)_{x x}=\left(V_{m}^{(\epsilon)}\right)_{u_{m}^{+}} \dot{u}_{m}^{+}+\left(V_{m}^{(\epsilon)}\right)_{u_{m}^{-}} \dot{u}_{m}^{-},
$$

where $\left(V_{m}^{(\epsilon)}\right)_{u_{m}^{ \pm}}$indicate the partial derivatives for $V^{(\epsilon)}$ with respect to the parameters $u_{m}^{ \pm}$, respectively. Since $V_{m}^{(\epsilon)} \rightarrow H\left(x-X_{m} ; u_{m}^{+}, u_{m}^{-}\right)$as $\epsilon \rightarrow 0+($ see $(2.10))$, it is expected that the right-hand side of $(2.11)$ approaches $H\left(x-X_{m}(t) ; \dot{u}_{m}^{+}, \dot{u}_{m}^{-}\right)$as $\epsilon$ is sufficiently small.

Lemma 2.3. Let $V^{(\epsilon)}\left(\zeta ; u_{m}^{+}, u_{m}^{-}\right)$be defined by that in Lemma 2.2. We have

$$
\begin{aligned}
& \left\|\left(V^{(\epsilon)}\right)_{u_{m}^{+}}\left(\bullet ; u_{m}^{+}, u_{m}^{-}\right) \dot{u}_{m}^{+}+\left(V^{(\epsilon)}\right)_{u_{m}^{-}}\left(\bullet ; u_{m}^{+}, u_{m}^{-}\right) \dot{u}_{m}^{-}-H\left(\bullet ; \dot{u}_{m}^{+}, \dot{u}_{m}^{-}\right)\right\| \\
\leq & C \epsilon\left(\left|u_{x}\left(X_{m}+0, t\right)\right|+\left|u_{x}\left(X_{m}-0, t\right)\right|\right),
\end{aligned}
$$

where $\dot{u}_{m}^{ \pm}=d\left(u_{m}^{ \pm}\right) / d t$.

We defer the proofs of the above two lemmas to the Appendix. 


\section{MAIN RESUlt AND OUtLine OF PROOF}

In the preceding sections we were concerned with the solution structure, traveling wave solution and stability lemma. Using these results, we are now ready to state and prove the main result of this paper.

Theorem 3.1. Let the flux $f$ be strictly convex. Assume that the initial data $u_{0}$ satisfies the requirements (A1)-(A4) stated in Section 1 and that a $\left(u_{0}\right)$ is sufficiently smooth near its negative minimum points. If $v_{\epsilon}$ is the solution to (0.3) and (0.4) and $u$ is the solution to (0.1) and (0.2), then the following error estimate holds:

$$
\sup _{0 \leq t \leq T}\left\|v_{\epsilon}(\bullet, t)-u(\bullet, t)\right\| \leq C(T)(\epsilon|\log \epsilon|+\epsilon),
$$

where $C(T)$ is a constant independent of $\epsilon$.

Before giving a proof for the above estimate, we point out a direct application of Theorem 3.1.

Corollary 3.1. Let the flux $f$ be strictly convex and $u_{0} \in C_{0}^{2}(\mathbf{R})$. If $a\left(u_{0}\right)$ has a finite number of inflection points and is sufficiently smooth at the decreasing inflection points, then the following error estimate holds:

$$
\sup _{0 \leq t \leq T}\left\|v_{\epsilon}(\bullet, t)-u(\bullet, t)\right\| \leq C(T)(\epsilon|\log \epsilon|+\epsilon) .
$$

Remark 3.1. Loosely speaking, the above result implies that for scalar convex conservation laws, if the initial data $u_{0}$ is smooth and $f^{\prime}\left(u_{0}\right)$ has a finite number of inflection points, then the $L^{1}$-convergence rate of viscosity methods is $O(\epsilon|\log \epsilon|)$. The class of initial data described in Corollary 3.1 is quite general, and is widely used in practical computations.

We will use Lemma 2.1 to prove Theorem 3.1. An essential requirement in Lemma 2.1 is that the solution to (2.1) should be continuous. It is known that the solution of $(0.3)$ is continuous for $t>0$ if the initial data is piecewise smooth. However, the solution of (0.1) may be discontinuous for $t>0$ even if the initial data is smooth. Therefore, we cannot apply Lemma 2.1 directly to equations (0.1) and (0.3).

As discussed before, there are two kinds of discontinuity, namely rarefaction wave and shock wave for solutions of (0.1) and (0.2). Across the rarefaction region, the solution is continuous, but on the shock curves, the solution is discontinuous. We need to construct a reasonable approximation to $u$ so that it can get rid of the shock discontinuities.

We will consider the cases when $u$ possesses shock discontinuity. For any interval $\left(t_{p}, t_{p+1}\right), 0 \leq p \leq P$ (here $t_{0}=0, t_{P+1}=T$ ), we will construct $\bar{v}_{\epsilon, p}(x, t)$, an approximate function to $u(x, t)$, such that

- (B1): $\bar{v}_{\epsilon, p}(x, t) \in C\left(\mathbf{R} \times\left(t_{p}, t_{p+1}\right)\right), \bar{v}_{\epsilon, p}(x, t)-v_{\epsilon} \rightarrow 0$, as $x \rightarrow \pm \infty$;

- (B2): $\bar{v}_{\epsilon, p}(x, t)$ is piecewise smooth and satisfies

$$
\left(\bar{v}_{\epsilon, p}\right)_{t}+\left(f\left(\bar{v}_{\epsilon, p}\right)\right)_{x}-\epsilon\left(\bar{v}_{\epsilon, p}\right)_{x x}=\bar{g}(x, t)
$$

in its smooth regions; where the right-hand side function satisfies

$$
\int_{t_{p}+\epsilon}^{t_{p+1}-\epsilon}\|\bar{g}(\bullet, t)\| d t \leq C \epsilon|\log \epsilon|+C \epsilon ;
$$


- (B3): $u_{x}$ and $\left(\bar{v}_{\epsilon, p}\right)_{x}$ are discontinuous on the same curves, namely, they are discontinuous on $X_{m}(t), 1 \leq m \leq M_{p}$, for $(x, t) \in\left(\mathbf{R} \times\left(t_{p}, t_{p+1}\right)\right)$ (see Section 1 for the definition of $X_{m}$; here for ease of notation we ignore the superscript $p$ and $n$ ). Moreover,

$$
\begin{aligned}
& \sum_{m}\left|\left(\bar{v}_{\epsilon, p}\right)_{x}\left(X_{m}(t)+0, t\right)-\left(\bar{v}_{\epsilon, p}\right)_{x}\left(X_{m}(t)-0, t\right)\right| \\
= & \sum_{m}\left|u_{x}\left(X_{m}(t)+0, t\right)-u_{x}\left(X_{m}(t)-0, t\right)\right| ;
\end{aligned}
$$

- (B4): For $t \in\left(t_{p}, t_{p+1}\right)$,

$$
\left\|\bar{v}_{\epsilon, p}(\bullet, t)-u(\bullet, t)\right\| \leq C \epsilon .
$$

It is known that $v_{\epsilon}$, the solution to (0.3) and (0.4), belongs to $C^{1}\left(\mathbf{R} \times\left(t_{p}, t_{p+1}\right)\right)$. If $\bar{v}_{\epsilon, p}$ satisfies (B1), (B2) and (B3), using Lemma 2.1 gives

$$
\begin{aligned}
& \left\|v_{\epsilon}(\bullet, t)-\bar{v}_{\epsilon, p}(\bullet, t)\right\| \leq\left\|v_{\epsilon}\left(\bullet, t_{p}+\epsilon\right)-\bar{v}_{\epsilon, p}\left(\bullet, t_{p}+\epsilon\right)\right\|+\int_{t_{p}+\epsilon}^{t}\|\bar{g}(\bullet, \tau)\| d \tau \\
& \quad+\epsilon \sum_{m} \int_{t_{p}+\epsilon}^{t}\left|u_{x}\left(X_{m}(\tau)+0, \tau\right)-u_{x}\left(X_{m}(\tau)-0, \tau\right)\right| d \tau
\end{aligned}
$$

for $t \in\left(t_{p}+\epsilon, t_{p+1}-\epsilon\right)$. Using Lemmas 1.1, 1.2 and (3.4) gives

$$
\left\|v_{\epsilon}(\bullet, t)-\bar{v}_{\epsilon, p}(\bullet, t)\right\| \leq\left\|v_{\epsilon}\left(\bullet, t_{p}+\epsilon\right)-\bar{v}_{\epsilon, p}\left(\bullet, t_{p}+\epsilon\right)\right\|+C \epsilon|\log \epsilon|+C \epsilon,
$$

for $t \in\left(t_{p}+\epsilon, t_{p+1}-\epsilon\right)$. It follows from the above inequality and (B4) that

$$
\left\|v_{\epsilon}(\bullet, t)-u(\bullet, t)\right\| \leq\left\|v_{\epsilon}\left(\bullet, t_{p}+\epsilon\right)-u\left(\bullet, t_{p}+\epsilon\right)\right\|+C \epsilon|\log \epsilon|+C \epsilon,
$$

for $t \in\left(t_{p}+\epsilon, t_{p+1}-\epsilon\right)$. Since $u$ and $v_{\epsilon}$ satisfy the following stability results,

$$
\left\|u\left(\cdot, s_{1}\right)-u\left(\cdot, s_{2}\right)\right\| \leq C\left|s_{1}-s_{2}\right|, \quad\left\|v_{\epsilon}\left(\cdot, s_{1}\right)-v_{\epsilon}\left(\cdot, s_{2}\right)\right\| \leq C\left|s_{1}-s_{2}\right|,
$$

we obtain

$$
\left\|v_{\epsilon}(\bullet, t)-u(\bullet, t)\right\| \leq\left\|v_{\epsilon}\left(\bullet, t_{p}\right)-u\left(\bullet, t_{p}\right)\right\|+C \epsilon|\log \epsilon|+C \epsilon, \quad t \in\left[t_{p}, t_{p+1}\right] .
$$

Noting that the above inequality is true for all $0 \leq p \leq P$, we obtain that for any $t \in[0, T]$,

$$
\begin{aligned}
\left\|v_{\epsilon}(\bullet, t)-u(\bullet, t)\right\| & \leq\left\|v_{\epsilon}(\bullet, 0)-u(\bullet, 0)\right\|+C \epsilon|\log \epsilon|+C \epsilon \\
& =C \epsilon|\log \epsilon|+C \epsilon .
\end{aligned}
$$

To prove Theorem 3.1, what remains is to construct $\bar{v}_{\epsilon, p}, 0 \leq p \leq P$, satisfying (B1)-(B4). We will concentrate on $\left(t_{p}, t_{p+1}\right)$, one of the intervals in $\bigcup_{p=0}^{P}\left(t_{p}, t_{p+1}\right)$.

Remark 3.2. Once again we point out that the main difficulty in using Lemma 2.1 is that the solution $u$ may be discontinuous. This happens when $u$ has shock discontinuities. If the solution $u$ contains contact discontinuities only (i.e. rarefaction waves), the solution is still continuous and Lemma 2.1 can be used directly. Therefore, the construction of $\bar{v}_{\epsilon}$ is to remove shock discontinuities and no efforts need be made to remove the contact discontinuities.

For ease of notation, we denote $\bar{v}_{\epsilon, p}$ by $\bar{v}_{\epsilon}$ in the remainder of this section. 
3.1. Zero shock. In this case, no shock occurs in the domain $\mathbf{R} \times\left(t_{p}, t_{p+1}\right)$. We simply choose $\bar{v}_{\epsilon}=u$. Since there is no shock discontinuity for $u$, we have $\bar{v}_{\epsilon}=$ $u \in C\left(\mathbf{R} \times\left(t_{p}, t_{p+1}\right)\right)$. It is straightforward to verify that this $\bar{v}_{\epsilon}$ satisfies the requirements (B1), (B3) and (B4). It is also obvious that $\bar{g}(x, t)$ in (3.3) equals $-\epsilon u_{x x}$. Using Lemma 1.3 we can verify that this $\bar{g}$ satisfies (3.4). Therefore, the requirement (B2) is also satisfied.

3.2. One shock. In this case, we assume that $u$ is smooth in $\mathbf{R} \times\left(t_{p}, t_{p+1}\right)$, except on $x=X_{m}(t)$. Here the function $X_{m}(t)$ satisfies (2.5). As mentioned before, Lemma 2.1 cannot be applied directly since $u \notin C\left(\mathbf{R} \times\left(t_{p}, t_{p+1}\right)\right)$. In order to overcome the difficulty, we introduce the following approximate solution:

$$
\bar{v}_{\epsilon}(x, t)=u(x, t)+\left[V^{(\epsilon)}\left(x-X_{m} ; u_{m}^{+}, u_{m}^{-}\right)-H\left(x-X_{m} ; u_{m}^{+}, u_{m}^{-}\right)\right] .
$$

We will verify that this $\bar{v}_{\epsilon}$ satisfies the requirements (B1)-(B4). First, we observe that

$$
\bar{v}_{\epsilon}\left(X_{m}+0, t\right)-\bar{v}_{\epsilon}\left(X_{m}-0, t\right)=V^{(\epsilon)}\left(0+; u_{m}^{+}, u_{m}^{-}\right)-V^{(\epsilon)}\left(0-; u_{m}^{+}, u_{m}^{-}\right)=0,
$$

which indicates that $\bar{v}_{\epsilon} \in C\left(\mathbf{R} \times\left(t_{p}, t_{p+1}\right)\right)$. It is also easy to see that $\bar{v}_{\epsilon}-u \rightarrow 0$ as $x \rightarrow \pm \infty$, which implies that $\bar{v}_{\epsilon}-v_{\epsilon} \rightarrow 0$ as $x \rightarrow \pm \infty$. Hence, (B1) is true. We can also show that (B3) is true. Using Lemma 2.2 will give (B4). We now need to show that $\bar{v}_{\epsilon}$ satisfies (B2).

Proof of (B2). We denote $V^{(\epsilon)}\left(x-X_{m} ; u_{m}^{+}, u_{m}^{-}\right)$by $V_{m}^{(\epsilon)}$, and $H\left(x-X_{m} ; u_{m}^{+}, u_{m}^{-}\right)$ by $H_{m}$. It follows from (2.11) that

$$
\left(V_{m}^{(\epsilon)}\right)_{t}+f\left(V_{m}^{(\epsilon)}\right)_{x}-\epsilon\left(V_{m}^{(\epsilon)}\right)_{x x}=\left(V_{m}^{(\epsilon)}\right)_{u_{m}^{+}} \dot{u}_{m}^{+}+\left(V_{m}^{(\epsilon)}\right)_{u_{m}^{-}} \dot{u}_{m}^{-} .
$$

This, together with (0.1), gives

$$
\left(\bar{v}_{\epsilon}\right)_{t}+\left(f\left(\bar{v}_{\epsilon}\right)\right)_{x}-\epsilon\left(\bar{v}_{\epsilon}\right)_{x x}=\bar{g}(x, t), \quad t \in\left(t_{p}, t_{p+1}\right),
$$

where

$$
\begin{aligned}
& \bar{g}(x, t)=I_{1}+I_{2}-\epsilon u_{x x}, \quad I_{1}=-(f(u))_{x}-f\left(V_{m}^{(\epsilon)}\right)_{x}+f\left(\bar{v}_{\epsilon}\right)_{x}, \\
& I_{2}=\left(V_{m}^{(\epsilon)}\right)_{u_{m}^{+}} \dot{u}_{m}^{+}+\left(V_{m}^{(\epsilon)}\right)_{u_{m}^{-}} \dot{u}_{m}^{-}-H\left(x-X_{m}(t) ; \dot{u}_{m}^{+}, \dot{u}_{m}^{-}\right) .
\end{aligned}
$$

Notice that $\left[H\left(x-X_{m}(t) ; u_{m}^{+}, u_{m}^{-}\right)\right]_{x}=0$ for all $x$ except on the curve $x=X_{m}(t)$. Away from $x=X_{m}(t)$, we have

$$
\begin{aligned}
I_{1} & =f^{\prime}\left(\bar{v}_{\epsilon}\right)\left(u_{x}+\left(V_{m}^{(\epsilon)}\right)_{x}\right)-f^{\prime}(u) u_{x}-f^{\prime}\left(V_{m}^{(\epsilon)}\right)\left(V_{m}^{(\epsilon)}\right)_{x} \\
& =\left(f^{\prime}\left(\bar{v}_{\epsilon}\right)-f^{\prime}(u)\right) u_{x}+\left(f^{\prime}\left(\bar{v}_{\epsilon}\right)-f^{\prime}\left(V_{m}^{(\epsilon)}\right)\right)\left(V_{m}^{(\epsilon)}\right)_{x} \\
& =f^{\prime \prime}\left(\xi_{1}\right)\left(V_{m}^{(\epsilon)}-H_{m}\right) u_{x}+f^{\prime \prime}\left(\xi_{2}\right)\left(u-H_{m}\right)\left(V_{m}^{(\epsilon)}\right)_{x} \\
& =: I_{1}^{(1)}+I_{1}^{(2)},
\end{aligned}
$$

where $\xi_{1}$ and $\xi_{2}$ are some intermediate values. For $x>X_{m}(t)$, although there are no shock curves across the interval $\left(X_{m}, x\right)$, there may exist a finite number of central rarefaction waves in the interval. Let $x=Y_{l}(t), 1 \leq l \leq L$, denote left 
or right boundaries of the rarefaction waves for $t \in\left(t_{p}, t_{p+1}\right)$. If $x>X_{m}(t)$ but $x \neq Y_{l}(t)$,

$u_{x}(x, t)=u_{x}\left(X_{m}(t)+0, t\right)+\sum_{l}\left(u_{x}\left(Y_{l}(t)+0, t\right)-u_{x}\left(Y_{l}(t)-0, t\right)\right)+\int_{X_{m}}^{x} u_{x x}(x, t) d x$.

It follows from the above result that, for $x>X_{m}(t)$,

$$
\left|u_{x}(x, t)\right| \leq\left|u_{x}\left(X_{m}(t)+0, t\right)\right|+\sum_{l}\left|u_{x}\left(Y_{l}(t) \pm 0, t\right)\right|+\left\|u_{x x}(\bullet, t)\right\| .
$$

Similarly, we can show that, for $x<X_{m}(t)$,

$$
\left|u_{x}(x, t)\right| \leq\left|u_{x}\left(X_{m}(t)-0, t\right)\right|+\sum_{l}\left|u_{x}\left(Y_{l}(t) \pm 0, t\right)\right|+\left\|u_{x x}(\bullet, t)\right\| .
$$

The above results and (2.10) lead to

$$
\left\|I_{1}^{(1)}(\bullet, t)\right\| \leq C \epsilon\left(\left|u_{x}\left(X_{m}(t) \pm 0, t\right)\right|+\sum_{l}\left|u_{x}\left(Y_{l}(t) \pm 0, t\right)\right|+\left\|u_{x x}(\bullet, t)\right\|\right) .
$$

An application of Lemmas 1.1, 1.2 and 1.3 yields

$$
\int_{t_{p}+\epsilon}^{t_{p+1}-\epsilon}\left\|I_{1}^{(1)}(\bullet, t)\right\| d t \leq C \epsilon|\log \epsilon|+C \epsilon .
$$

Using the facts that $\left[H\left(x-X_{m}(t) ; u_{m}^{+}, u_{m}^{-}\right)\right]_{x}=0$, a.e. and $\left(V^{(\epsilon)}\right)_{x} \leq 0$, we have

$$
\left\|I_{1}^{(2)}(\bullet, t)\right\| \leq C \int_{-\infty}^{\infty}\left|u-H_{m}\right|\left(H_{m}-V_{m}^{(\epsilon)}\right)_{x} d x .
$$

For any $x \neq X_{m}(t)$, we can show that

$$
\left|u(x, t)-H_{m}\right| \leq \max _{z \in \mathbf{R}}\left|u_{x}(z \pm 0, t)\right|\left|x-X_{m}(t)\right| .
$$

For $x \neq X_{m}(t)$, using (3.8) and (3.9) yields

$$
\left|u(x, t)-H_{m}\right| \leq\left(\left|u_{x}\left(X_{m}(t) \pm 0, t\right)\right|+\sum_{l}\left|u_{x}\left(Y_{l}(t) \pm 0, t\right)\right|+\left\|u_{x x}(\bullet, t)\right\|\right)\left|x-X_{m}(t)\right| .
$$

Hence, from (3.10) we have

$$
\begin{aligned}
\left\|I_{1}^{(2)}(\bullet, t)\right\| \leq C & \left(\left|u_{x}\left(X_{m}(t) \pm 0, t\right)\right|+\sum_{l}\left|u_{x}\left(Y_{l}(t) \pm 0, t\right)\right|+\left\|u_{x x}(\bullet, t)\right\|\right) \\
& \times \int_{-\infty}^{\infty}\left|x-X_{m}(t)\right|\left(H_{m}-V_{m}^{(\epsilon)}\right)_{x} d x .
\end{aligned}
$$

Using integration by parts and Lemma 2.2, we can show that the integral on the right-hand side can be bounded by $C \epsilon$. Further, using Lemmas 1.1, 1.2 and 1.3 gives

$$
\int_{t_{p}+\epsilon}^{t_{p+1}-\epsilon}\left\|I_{1}^{(2)}(\bullet, t)\right\| d t \leq C \epsilon|\log \epsilon|+C \epsilon
$$


We have therefore obtained an estimate for $I_{1}$. An application of Lemmas 1.1 and 2.3 yields

$$
\int_{t_{p}+\epsilon}^{t_{p+1}-\epsilon}\left\|I_{2}(\bullet, t)\right\| d t \leq C \epsilon|\log \epsilon|+C \epsilon .
$$

Using the estimates for $I_{1}$ and $I_{2}$, together with Lemma 1.3, we establish the inequality (3.4).

Remark 3.3. From the definition of $\bar{g}(x, t)$, we find that it is necessary to estimate $u_{x x}$. Therefore, Lemma 1.3 is useful in obtaining our error estimates.

3.3. Two shocks. In this case, we have two smooth shock curves, $X_{m}(t)$ and $X_{m+1}(t)$, satisfying one of the following possibilities:

- (P1): $X_{m+1}(t)>X_{m}(t)$ for $t \in\left[t_{p}, t_{p+1}\right]$, see Case H in Figure 1 ;

- (P2): $X_{m+1}(t)>X_{m}(t)$ for $t \in\left[t_{p}, t_{p+1}\right)$ and $X_{m}\left(t_{p+1}\right)=X_{m+1}\left(t_{p+1}\right)$, see Case D in Figure 1;

- (P3): there exists a time level $t^{(p, 1)} \in\left(t_{p}, t_{p+1}\right)$ such that there are two shock waves for $t \in\left(t_{p}, t^{(p, 1)}\right)$, but the two shocks meet at $t=t^{(p, 1)}$ and form one shock for $t \in\left[t^{(p, 1)}, t_{p+1}\right)$, see Cases C and D in Figure 1 .

For the first two possibilities, we introduce the following approximate solution:

$$
\begin{aligned}
& \bar{v}_{\epsilon}(x, t)=u(x, t)+\left[V^{(\epsilon)}\left(x-X_{m}(t) ; u_{m}^{+}, u_{m}^{-}\right)-H\left(x-X_{m}(t) ; u_{m}^{+}, u_{m}^{-}\right)\right] \\
& \quad+\left[V^{(\epsilon)}\left(x-X_{m+1}(t) ; u_{m+1}^{+}, u_{m+1}^{-}\right)-H\left(x-X_{m+1}(t) ; u_{m+1}^{+}, u_{m+1}^{-}\right)\right] .
\end{aligned}
$$

We can verify that $\bar{v}_{\epsilon}$ satisfies the requirements (B1), (B3) and (B4). Again, we will show that $\bar{v}_{\epsilon}$ satisfies (B2).

Proof of (B2). We denote $V^{(\epsilon)}\left(x-X_{m}(t) ; u_{m}^{+}, u_{m}^{-}\right)$and $H\left(x-X_{m}(t) ; u_{m}^{+}, u_{m}^{-}\right)$by $V_{m}^{(\epsilon)}$ and $H_{m}$, respectively. It follows from (2.11) that

$$
\begin{aligned}
& \left(V_{m}^{(\epsilon)}\right)_{t}+f\left(V_{m}^{(\epsilon)}\right)_{x}-\epsilon\left(V_{m}^{(\epsilon)}\right)_{x x}+\left(V_{m+1}^{(\epsilon)}\right)_{t}+f\left(V_{m+1}^{(\epsilon)}\right)_{x}-\epsilon\left(V_{m+1}^{(\epsilon)}\right)_{x x} \\
= & \left(V_{m}^{(\epsilon)}\right)_{u_{m}^{+}} \dot{u}_{m}^{+}+\left(V_{m}^{(\epsilon)}\right)_{u_{m}^{-}} \dot{u}_{m}^{-}+\left(V_{m+1}^{(\epsilon)}\right)_{u_{m+1}^{+}} \dot{u}_{m+1}^{+}+\left(V_{m+1}^{(\epsilon)}\right)_{u_{m+1}^{-}} \dot{u}_{m+1}^{-} .
\end{aligned}
$$

This, together with (0.1), gives

$$
\left(\bar{v}_{\epsilon}\right)_{t}+f\left(\bar{v}_{\epsilon}\right)_{x}-\epsilon\left(\bar{v}_{\epsilon}\right)_{x x}=\bar{g}(x, t), \quad t \in\left(t_{p}, t_{p+1}\right),
$$

where

$$
\begin{aligned}
& \bar{g}(x, t)=I_{1}+I_{2}-\epsilon u_{x x} \\
& I_{1}=-f(u)_{x}-f\left(V_{m}^{(\epsilon)}\right)_{x}-f\left(V_{m+1}^{(\epsilon)}\right)_{x}+f\left(\bar{v}_{\epsilon}\right)_{x}, \\
& I_{2}=\left(V_{m}^{(\epsilon)}\right)_{u_{m}^{+}} \dot{u}_{m}^{+}+\left(V_{m}^{(\epsilon)}\right)_{u_{m}^{-}} \dot{u}_{m}^{-}-H\left(x-X_{m}(t) ; \dot{u}_{m}^{+}, \dot{u}_{m}^{-}\right) \\
& \quad+\left(V_{m+1}^{(\epsilon)}\right)_{u_{m+1}^{+}} \dot{u}_{m+1}^{+}+\left(V_{m+1}^{(\epsilon)}\right)_{u_{m+1}^{-}} \dot{u}_{m+1}^{-} \\
& \quad-H\left(x-X_{m+1}(t) ; \dot{u}_{m+1}^{+}, \dot{u}_{m+1}^{-}\right) .
\end{aligned}
$$


We only need to estimate $\left\|I_{1}\right\|$; other terms on the right-hand side of (3.12) can be handled by Lemmas 2.3, 1.1, 1.2 and 1.3. Observe that

$$
\begin{aligned}
I_{1}= & f^{\prime}\left(\bar{v}_{\epsilon}\right)\left(u_{x}+\left(V_{m}^{(\epsilon)}\right)_{x}+\left(V_{m+1}^{(\epsilon)}\right)_{x}\right)-f^{\prime}(u) u_{x} \\
& \quad-f^{\prime}\left(V_{m}^{(\epsilon)}\right)\left(V_{m}^{(\epsilon)}\right)_{x}-f^{\prime}\left(V_{m+1}^{(\epsilon)}\right)\left(V_{m+1}^{(\epsilon)}\right)_{x} \\
= & f^{\prime \prime}\left(\xi_{1}\right)\left(\bar{v}_{\epsilon}-u\right) u_{x}+f^{\prime \prime}\left(\xi_{2}\right)\left(\bar{v}_{\epsilon}-V_{m}^{(\epsilon)}\right)\left(V_{m}^{(\epsilon)}\right)_{x} \\
& \quad+f^{\prime \prime}\left(\xi_{3}\right)\left(\bar{v}_{\epsilon}-V_{m+1}^{(\epsilon)}\right)\left(V_{m+1}^{(\epsilon)}\right)_{x} \\
= & I_{1}^{(1)}+I_{1}^{(2)}+I_{1}^{(3)},
\end{aligned}
$$

where $\xi_{1}, \xi_{2}, \xi_{3}$ are some intermediate values. Similar to the proof of (3.8) and (3.9), we have

$$
\begin{aligned}
\left|u_{x}(x \pm 0, t)\right| \leq \mid & u_{x}\left(X_{m} \pm 0, t\right)|+| u_{x}\left(X_{m+1} \pm 0, t\right) \mid \\
& +\sum_{l}\left|u_{x}\left(Y_{l}(t) \pm 0, t\right)\right|+\left\|u_{x x}(\bullet, t)\right\|,
\end{aligned}
$$

for any $(x, t) \in \mathbf{R} \times\left(t_{p}, t_{p+1}\right)$, where $x=Y_{l}(t), 1 \leq l \leq L$, denotes left or right boundaries of (possible) rarefaction waves. This, together with Lemma 2.2 (more precisely (2.10)), leads to

$$
\int_{t_{p}+\epsilon}^{t_{p+1}-\epsilon}\left\|I_{1}^{(1)}(\bullet, t)\right\| d t \leq C \epsilon|\log \epsilon|+C \epsilon .
$$

Observe that

$$
\begin{aligned}
\left|I_{1}^{(2)}\right| & \leq C\left|u-H_{m}\right|\left(-V_{m}^{(\epsilon)}\right)_{x}+C\left|V_{m+1}^{(\epsilon)}-H_{m+1}\right|\left(-V_{m}^{(\epsilon)}\right)_{x} \\
& =: J_{1}+J_{2}
\end{aligned}
$$

From (2.9) we have

$$
\begin{aligned}
& \left\|J_{2}(\bullet, t)\right\|=C \int_{-\infty}^{\infty}\left|V_{m+1}^{(\epsilon)}-H_{m+1}\right|\left(-V_{m}^{(\epsilon)}\right)_{x} d x \\
\leq & C \beta_{m+1} \int_{-\infty}^{\infty} \exp \left(-\beta_{m+1} \frac{\left|x-X_{m+1}(t)\right|}{2 \epsilon}\right)\left(-V_{m}^{(\epsilon)}\right)_{x} d x \\
= & C \beta_{m+1}\left[\int_{\left|x-X_{m+1}(t)\right| \leq \delta(t) / 2}+\int_{\left|x-X_{m+1}(t)\right| \geq \delta(t) / 2}\right] \\
\leq & C \beta_{m+1}\left[\int_{\left|x-X_{m+1}\right| \leq \delta / 2}\left(-V_{m}^{(\epsilon)}\right)_{x} d x+\exp \left(-\beta_{m+1} \frac{\delta(t)}{4 \epsilon}\right) \int_{-\infty}^{\infty}\left(-V_{m}^{(\epsilon)}\right)_{x} d x\right] \\
\leq & C \beta_{m+1}\left[V\left(\frac{\delta(t)}{2 \epsilon} ; u_{m}^{+}, u_{m}^{-}\right)-V\left(\frac{3 \delta(t)}{2 \epsilon} ; u_{m}^{+}, u_{m}^{-}\right)+\beta_{m} \exp \left(-\beta_{m+1} \frac{\delta(t)}{4 \epsilon}\right)\right],
\end{aligned}
$$

where $\beta_{m}=u_{m}^{-}-u_{m}^{+}, \beta_{m+1}=u_{m+1}^{-}-u_{m+1}^{+}, \delta(t)=X_{m+1}(t)-X_{m}(t)$. It follows from (2.9) that

$$
\begin{aligned}
& V\left(\frac{\delta(t)}{2 \epsilon} ; u_{m}^{+}, u_{m}^{-}\right)-V\left(\frac{3 \delta(t)}{2 \epsilon} ; u_{m}^{+}, u_{m}^{-}\right) \\
\leq & V\left(\frac{\delta(t)}{2 \epsilon} ; u_{m}^{+}, u_{m}^{-}\right)-u_{m}^{+} \leq \beta_{m} \exp \left(-\beta_{m} \frac{\delta(t)}{4 \epsilon}\right) .
\end{aligned}
$$


Consequently, we obtain

$$
\left\|J_{2}(\bullet, t)\right\| \leq C \beta_{m+1} \beta_{m}\left\{\exp \left(-\beta_{m} \frac{\delta(t)}{4 \epsilon}\right)+\exp \left(-\beta_{m+1} \frac{\delta(t)}{4 \epsilon}\right)\right\} .
$$

Consider the following function:

$$
z(t)=\frac{t_{p+1}-t}{X_{m+1}(t)-X_{m}(t)}, \quad t \in\left[t_{p}, t_{p+1}\right] .
$$

It is obvious that $z(t) \in C\left[t_{p}, t_{p+1}\right)$. For the first possibility, (P1), $X_{m+1}(t)-$ $X_{m}(t)>0$ for all $t \in\left[t_{p}, t_{p+1}\right]$, which indicates that $z \in C\left[t_{p}, t_{p+1}\right]$. For the second possibility, (P2), we have $u_{m}^{+}\left(t_{p+1}\right)=u_{m+1}^{-}\left(t_{p+1}\right)$. It follows from the definition (2.5) that

$$
\dot{X}_{m}\left(t_{p+1}\right)-\dot{X}_{m+1}\left(t_{p+1}\right)=\int_{0}^{1} f^{\prime \prime}(\xi) \theta d \theta\left(u_{m}^{-}\left(t_{p+1}\right)-u_{m+1}^{+}\left(t_{p+1}\right)\right),
$$

where $\xi$ is an intermediate value. Since $X_{m+1}\left(t_{p+1}\right)=X_{m}\left(t_{p+1}\right)$, we have

$$
\begin{aligned}
0 & <\zeta_{m+1}^{+}\left(t_{p+1}\right)-\zeta_{m}^{-}\left(t_{p+1}\right)=t_{p+1}\left(a\left(u_{m}^{-}\left(t_{p+1}\right)\right)-a\left(u_{m+1}^{+}\left(t_{p+1}\right)\right)\right) \\
& \leq C\left(u_{m}^{-}\left(t_{p+1}\right)-u_{m+1}^{+}\left(t_{p+1}\right)\right) .
\end{aligned}
$$

This and (3.16) lead to

$$
\dot{X}_{m}\left(t_{p+1}\right)-\dot{X}_{m+1}\left(t_{p+1}\right) \geq C>0 .
$$

From this and L'Hospital's rule, we have

$$
\lim _{t \rightarrow t_{p+1}-0} z(t)=\frac{1}{\dot{X}_{m}\left(t_{p+1}\right)-\dot{X}_{m+1}\left(t_{p+1}\right)} \leq C .
$$

This indicates that $z \in C\left[t_{p}, t_{p+1}\right]$. Therefore, for both possibilities (P1) and (P2), there exists a constant $\gamma>0$, such that $|z(t)| \leq \gamma$ for all $t \in\left[t_{p}, t_{p+1}\right]$. It follows from the definition of $z(t)$ that

$$
\delta(t)=X_{m+1}(t)-X_{m}(t) \geq C\left(t_{p+1}-t\right), \quad t_{p} \leq t \leq t_{p+1} .
$$

From this and (3.15), we have

$$
\left\|J_{2}(\bullet, t)\right\| \leq C \beta_{m+1} \beta_{m}\left\{\exp \left(-C \beta_{m+1}\left(t_{p+1}-t\right) / \epsilon\right)+\exp \left(-C \beta_{m}\left(t_{p+1}-t\right) / \epsilon\right)\right\} .
$$

This yields

$$
\int_{t_{p}}^{t_{p+1}}\left\|J_{2}(\bullet, t)\right\| d t \leq C \epsilon
$$

The estimate for $J_{1}$ is a little simpler than that for $J_{2}$. It follows from the facts

$$
\left|u(x, t)-H_{m}\right| \leq\left\{\begin{array}{lll}
\max _{z<X_{m+1}(t)}\left|u_{x}(z \pm 0, t)\right|\left|x-X_{m}(t)\right|, & \text { if } \quad x<X_{m+1}(t), \\
C, & \text { if } \quad x \geq X_{m+1}(t),
\end{array}\right.
$$


that

$$
\begin{gathered}
\left\|J_{1}(\bullet, t)\right\|=C \int_{-\infty}^{\infty}\left|u-H_{m}\right|\left(-V_{m}^{(\epsilon)}\right)_{x} d x \\
\leq \quad C \max _{z<X_{m+1}(t)}\left|u_{x}(z \pm 0, t)\right| \int_{-\infty}^{X_{m+1}}\left|x-X_{m}(t)\right|\left(-V_{m}^{(\epsilon)}\right)_{x} d x \\
\quad+C \int_{X_{m+1}}^{\infty}\left(-V_{m}^{(\epsilon)}\right)_{x} d x \\
\leq \quad \max _{z<X_{m+1}(t)}\left|u_{x}(z \pm 0, t)\right| \int_{-\infty}^{\infty}\left|x-X_{m}(t)\right|\left(H_{m}-\left(-V_{m}^{(\epsilon)}\right)\right)_{x} d x \\
\quad+C\left(V\left(\delta(t) / \epsilon ; u_{m}^{+}, u_{m}^{-}\right)-u_{m}^{+}\right) .
\end{gathered}
$$

Using integration by parts and Lemma 2.2 , we can show that the last integral can be bounded by $C \epsilon$. Similar to the proof for $J_{2}$, we have

$$
0<V\left(\delta(t) / \epsilon ; u_{m}^{+}, u_{m}^{-}\right)-u_{m}^{+} \leq \beta_{m} \exp \left(-C \beta_{m}\left(t_{p+1}-t\right) / \epsilon\right) .
$$

These results, together with (3.13), yield

$$
\int_{t_{p}+\epsilon}^{t_{p+1}-\epsilon}\left\|J_{1}(\bullet, t)\right\| d t \leq C \epsilon|\log \epsilon|+C \epsilon
$$

Therefore, for both possibilities (P1) and (P2), we have

$$
\int_{t_{p}+\epsilon}^{t_{p+1}-\epsilon}\left\|I_{1}^{(2)}(\bullet, t)\right\| d t \leq C|\log \epsilon|+C \epsilon
$$

In a similar manner we can show that the above estimate holds also for $I_{1}^{(3)}$. Hence, we have proved that in cases (P1) and (P2) the function $\bar{v}_{\epsilon}$ given by (3.11) satisfied the requirement (B2).

If the possibility (P3) is the case, namely there are two shock curves, $X_{m}(t)$ and $X_{m+1}(t)$, for $t \in\left(t_{p}, t^{(p, 1)}\right)$, and one shock, $X_{m}(t)$, for $t \in\left(t^{(p, 1)}, t_{p+1}\right)$, we construct $\bar{v}_{\epsilon}$ in the following way:

$$
\bar{v}_{\epsilon}= \begin{cases}u(x, t)+\left(V_{m}^{(\epsilon)}-H_{m}\right)+\left(V_{m+1}^{(\epsilon)}-H_{m+1}\right), & \text { if } t \in\left(t_{p}, t^{(p, 1)}\right), \\ u(x, t)+\left(V_{m}^{(\epsilon)}-H_{m}\right), & \text { if } t \in\left[t^{(p, 1)}, t_{p+1}\right) .\end{cases}
$$

This case can be regarded as a combination of possibility (P2) analyzed above and the one shock case analyzed in the last subsection. It is then not difficult to verify that this $\bar{v}_{\epsilon}$ satisfies the requirements (B1)-(B4).

3.4. More shocks. Assume that we have $M$ shock curves $X_{m}(t), 1 \leq m \leq M$ for $t \in\left(t_{p}, t^{(p, 1)}\right)$, where $t^{(p, 1)} \leq t_{p+1}$. These shocks may emerge and there will be a lesser number of shocks for $t \in\left(t^{(p, 1)}, t_{p+1}\right)$. Similar to the last two subsections, we let

$$
\bar{v}_{\epsilon}(x, t)=u(x, t)+\sum_{m=1}^{M}\left(V^{(\epsilon)}\left(x-X_{m}(t) ; u_{m}^{+}, u_{m}^{-}\right)-H\left(x-X_{m}(t) ; u_{m}^{+}, u_{m}^{-}\right)\right),
$$

for $(x, t) \in \mathbf{R} \times\left(t_{p}, t^{(p, 1)}\right)$. We found that it is straightforward to extend the method of proof in the last subsection to show that the function $\bar{v}_{\epsilon}$ given by (3.18) satisfies 
the requirements (B1)-(B4). It is found that the following estimates are particularly useful:

$$
\begin{aligned}
&(3.19)\left\|\bar{v}_{\epsilon}(\bullet, t)-u(\bullet, t)\right\| \leq \sum_{m=1}^{M}\left\|V_{m}^{(\epsilon)}-H_{m}\right\| \leq C \epsilon \\
&(3.20)\left|u_{x}(x \pm 0, t)\right| \leq \sum_{m=1}^{M}\left|u_{x}\left(X_{m}(t) \pm 0, t\right)\right|+\sum_{l}\left|u_{x}\left(Y_{l}(t) \pm 0, t\right)\right|+\left\|u_{x x}(\bullet, t)\right\| ; \\
&(3.21) \|\left\|\left(\bar{v}_{\epsilon}(\bullet, t)-V_{k}^{(\epsilon)}\right)\left(V_{k}^{(\epsilon)}\right)_{x}\right\| \\
& \leq\left\|\left(u(\bullet, t)-H_{k}\right)\left(V_{k}^{(\epsilon)}\right)_{x}\right\|+\sum_{m \neq k}\left\|\left(V_{m}^{(\epsilon)}-H_{m}\right)\left(V_{k}^{(\epsilon)}\right)_{x}\right\| \\
& \leq \max _{X_{k-1}<z<X_{k+1}}\left|u_{x}(z \pm 0, t)\right| C \epsilon+C \beta_{k} \exp \left(-C \beta_{k}\left(t_{p+1}-t\right) / \epsilon\right) \\
&+\sum_{m \neq k} \beta_{m} \beta_{k}\left\{\exp \left(-C \beta_{m}\left(t_{p+1}-t\right) / \epsilon\right)+\exp \left(-C \beta_{k}\left(t_{p+1}-t\right) / \epsilon\right)\right\}
\end{aligned}
$$

for $1 \leq k \leq M$, where $\beta_{m}=u_{m}^{-}-u_{m}^{+}, \beta_{k}=u_{k}^{-}-u_{k}^{+}$. Here we have set $X_{0}(t)=$ $-\infty, X_{M+1}(t)=\infty$.

For $t \in\left[t^{(p, 1)}, t_{p+1}\right)$, the number of shock curves is less than $M$ and we may use mathematical induction (based on the number of shock curves) to show that an appropriate $\bar{v}_{\epsilon}$ can be found (cf. [20]). Therefore, Theorem 3.1 is proved.

Remark 3.4. Under the present framework, the factor $O(|\log \epsilon|)$ cannot be removed from our results. We found the factor is due to the estimates for $u_{x}$ and $u_{x x}$, see Lemmas 1.1 to 1.3. In some special cases, these estimates can be improved so that optimal convergence rates can be obtained.

\section{Remarks on SharP estimate}

In this section, we consider the case when the initial function $u_{0}$ satisfies the following conditions:

- (C1): $u_{0}(x)$ is bounded and piecewise $C^{2}$-smooth with a finite number of discontinuous points $\gamma_{i}, 1 \leq i \leq I ; \dot{u}_{0}\left(\gamma_{i} \pm 0\right)$ exist and are finite; $u_{0}\left(\gamma_{i}-0\right)>$ $u_{0}\left(\gamma_{i}+0\right)$;

- $(\mathrm{C} 2): \ddot{u}_{0} \in L^{1}(\mathbf{R})$;

- (C3):

$$
\lim _{|x| \rightarrow \infty} \dot{a}\left(u_{0}(x)\right)=0
$$

- (C4): $a\left(u_{0}\right)$ has no decreasing inflection points, but may have a finite number of increasing inflection points.

The assumption (C4) implies that there is no original shock generated after $t=0$. The assumption $(\mathrm{C} 1)$ implies that there is no central rarefaction waves formed at $t=0$. Therefore, only discontinuities are initial shocks and the interactions of the initial shocks. Assume $x=X(t)$ is a shock curve. Following Lemma 1.1, we can show that

$$
\begin{aligned}
& \int_{0}^{T}\left|u_{x}(X(t) \pm 0, t)\right| d t \leq C, \\
& \int_{0}^{T} \frac{1}{1+\dot{a}\left(u_{0}\left(\zeta^{ \pm}\right)\right) t} d t \leq C .
\end{aligned}
$$


We now use notations introduced in Section 1 . The interval $[0, T]$ can be divided into several subintervals: $0=t^{(0)}<t^{(1)}<\cdots<t^{(N)}=T$. In each interval $\left[t^{(n)}, t^{(n+1)}\right)$, there are a finite number of smooth curves $x=X_{m}^{(n)}(t)$ satisfying

$$
X_{m}^{(n)}(t)<X_{m+1}^{(n)}(t), \quad m=1, \cdots, M_{n}-1 .
$$

From each point on $x=X_{m}^{(n)}(t)$, we can trace two characteristic lines back to $t=0$ : $X_{m}^{(n)}(t)=\zeta_{m}^{ \pm}+a\left(u_{0}\left(\zeta_{m}^{ \pm}\right)\right) t$. Using (1.29) and the assumption (C4) gives

$$
\frac{1}{1+\dot{a}\left(u_{0}(\zeta)\right) t} \leq 1+\frac{1}{1+\dot{a}\left(u_{0}\left(\zeta_{m}^{+}\right)\right) t}+\frac{1}{1+\dot{a}\left(u_{0}\left(\zeta_{m+1}^{-}\right)\right) t},
$$

for $\zeta_{m}^{+} \leq \zeta \leq \zeta_{m+1}^{-}$with $m=0, \cdots, M_{n}$, where we have set $\zeta_{0}^{+}=-\infty, \zeta_{M_{n}+1}^{-}=\infty$. It follows from (4.2) that the integral for the right-hand side functions over $[0, T]$ can be bounded by a constant $C$. Following the proof for Lemma 1.3 we can show that

$$
\int_{0}^{T}\left\|u_{x x}(\bullet, t)\right\| d t \leq C .
$$

Using (4.1)-(4.3) and repeating the procedures in the last section yield the following theorem.

Theorem 4.1. Let the flux $f$ be strictly convex. Assume that the initial data $u_{0}$ satisfies the requirements $(\mathrm{C} 1)-(\mathrm{C} 4)$ stated above. If $v_{\epsilon}$ is the solution to $(0.3)$ and (0.4) and $u$ is the solution to (0.1) and (0.2), then the following error estimate holds:

$$
\sup _{0 \leq t \leq T}\left\|v_{\epsilon}(\bullet, t)-u(\bullet, t)\right\| \leq C(T) \epsilon,
$$

where $C(T)$ is a constant independent of $\epsilon$.

It is easy to show that $O(\epsilon)$ convergence rate is the best possible one for viscosity methods. The above result indicates that if the solution to (0.1) and (0.2) includes neither central rarefaction waves nor spontaneous shocks, then viscosity methods yield the optimal convergence rate.

\section{RESUlts ON NONINCREASING INITIAL DATA}

Throughout this section, $c$ denotes a positive constant independent of $t$ and $\epsilon$, but with different values at different places. We consider a special case when the initial function satisfies the following conditions:

- (D1): $u_{0}(x)$ is bounded, nonincreasing and piecewise $C^{2}$ smooth with a finite number of discontinuous points $\gamma_{1}<\cdots<\gamma_{I} ; \dot{u}_{0}\left(\gamma_{i} \pm 0\right)$ exist and are bounded;

- (D2): $\ddot{u}_{0}(x)$ does not change sign when $|x| \geq \Lambda$, with $\Lambda$ a positive constant;

- (D3):

$$
\lim _{|x| \rightarrow \infty} \dot{a}\left(u_{0}(x)\right)=0 ;
$$

- (D4): $\ddot{a}\left(u_{0}\right)$ has no inflection points.

Since $\ddot{a}\left(u_{0}\right)$ does not change sign in each of its smooth domains, we obtain from (D1) and (D3) that $\ddot{a}\left(u_{0}\right) \in L^{1}(\mathbf{R})$. The assumption (D1) also indicates that $\dot{u}_{0}(x \pm 0)$ is bounded for $|x| \leq \Lambda$. Observe that

$$
\ddot{a}\left(u_{0}\right)=a^{\prime \prime}\left(u_{0}\right)\left(\dot{u}_{0}\right)^{2}+a^{\prime}\left(u_{0}\right) \ddot{u}_{0},
$$


where $a^{\prime}\left(u_{0}\right)=f^{\prime \prime}\left(u_{0}\right) \geq \alpha>0$. The above results yield that $\ddot{u}_{0} \in L^{1}(-\Lambda, \Lambda)$. This, together with (D2) and (D3), imply that $\ddot{u}_{0} \in L^{1}(\mathbf{R})$.

The assumption (D1) also indicates that $u_{0}\left(\gamma_{i}-0\right)>u_{0}\left(\gamma_{i}+0\right)$. Since $u_{0}$ is nonincreasing, it is known that there exists a time level $T^{*}$ such that there is only one shock after $t=T^{*}$. The solution is smooth away from this shock for $t>T^{*}$. From each point on the shock curve, we can trace two characteristic lines $X(t)=\zeta^{ \pm}+a\left(u_{0}\left(\zeta^{ \pm}\right)\right)$back to $t=0$. It is obvious that

$$
\zeta^{-}(t)<\gamma_{1}, \quad \zeta^{+}(t)>\gamma_{I}, \quad T^{*} \leq t<\infty .
$$

It follows from (D1) that

$$
u_{0}\left(\zeta^{-}\right) \geq u_{0}\left(\gamma_{1}-0\right), \quad u_{0}\left(\zeta^{+}\right) \leq u_{0}\left(\gamma_{I}+0\right), \quad T^{*} \leq t<\infty,
$$

which leads to

$$
\left|u_{0}\left(\zeta^{-}\right)-u_{0}\left(\zeta^{+}\right)\right| \geq u_{0}\left(\gamma_{1}-0\right)-u_{0}\left(\gamma_{I}+0\right)=\text { constant }>0,
$$

for $T^{*} \leq t<\infty$. From (1.2), (1.4) and (5.2), we have

$$
\left|\dot{\zeta}^{-}\right| \geq \frac{c}{1+\dot{a}\left(u_{0}\left(\zeta^{-}\right)\right) t} \geq c>0,
$$

where we have used the fact $\dot{a}\left(u_{0}\right) \leq 0$ (due to (D1)). Since $\dot{\zeta}^{-}<0$, we obtain that

$$
\lim _{t \rightarrow \infty} \zeta^{-}(t)=-\infty \text {. }
$$

Moreover, from (D1), (D3) and (D4), we have

$$
\ddot{a}\left(u_{0}(\zeta)\right) \leq 0, \quad \zeta \in\left(-\infty, \gamma_{1}\right), \quad \text { and } \quad \ddot{a}\left(u_{0}(\zeta)\right) \geq 0, \quad \zeta \in\left(\gamma_{I}, \infty\right) .
$$

We need to prove the following results for some finite values $T_{1}, T_{2}>T^{*}$ :

$$
\begin{aligned}
& \int_{T_{1}}^{\infty}\left|u_{x}(X(t)-0, t)\right| d t \leq c, \quad \int_{T_{2}}^{\infty}\left|u_{x}(X(t)+0, t)\right| d t \leq c ; \\
& \int_{T_{1}}^{\infty} \int_{-\infty}^{\zeta^{-}(t)} \frac{\left|\dot{u}_{0} \ddot{a}\left(u_{0}\right)\right| t}{\left(1+\dot{a}\left(u_{0}\right) t\right)^{2}} d \zeta d t \leq c, \quad \int_{T_{2}}^{\infty} \int_{\zeta^{+}(t)}^{\infty} \frac{\left|\dot{u}_{0} \ddot{a}\left(u_{0}\right)\right| t}{\left(1+\dot{a}\left(u_{0}\right) t\right)^{2}} d \zeta d t \leq c ; \\
& \int_{T_{1}}^{\infty} \int_{-\infty}^{\zeta^{-}(t)} \frac{\left|\ddot{u}_{0}\right|}{1+\dot{a}\left(u_{0}\right) t} d \zeta d t \leq c, \quad \int_{T_{2}}^{\infty} \int_{\zeta^{+}(t)}^{\infty} \frac{\left|\ddot{u}_{0}\right|}{1+\dot{a}\left(u_{0}\right) t} d \zeta d t \leq c .
\end{aligned}
$$

It follows from (5.4) that there exists a time level $t=T_{1}>T^{*}$ such that $\zeta^{-}(t) \leq$ $-\Lambda$ for $t \geq T_{1}$. From (1.14) and (5.3), we obtain

$$
\begin{aligned}
\int_{T_{1}}^{\infty}\left|u_{x}(X(t)-0, t)\right| d t & \leq c \int_{T_{1}}^{\infty}\left|\dot{\zeta}^{-}(t) \dot{u}_{0}\left(\zeta^{-}\right)\right| d t \\
& \leq c\left(u_{0}(-\infty)-u_{0}\left(\zeta^{-}\left(T_{1}\right)\right)\right) \leq c,
\end{aligned}
$$

where in the second inequality we have used the facts that $\dot{\zeta}^{-} \leq 0$ and $\dot{u}_{0}(\zeta) \leq 0$. Hence the first inequality in (5.6) is proved. Further, using (5.5) gives

$$
\int_{-\infty}^{\zeta^{-}(t)} \frac{\left|\ddot{a}\left(u_{0}\right)\right| t}{\left(1+\dot{a}\left(u_{0}\right) t\right)^{2}} d \zeta=\frac{1}{1+\dot{a}\left(u_{0}\left(\zeta^{-}\right)\right) t}-1 \leq \frac{1}{1+\dot{a}\left(u_{0}\left(\zeta^{-}\right)\right) t} .
$$

It follows from (D2) and (D3) that

$$
\left|\dot{u}_{0}(\zeta)\right| \leq\left|\dot{u}_{0}\left(\zeta^{-}\right)\right| \quad \text { for } \quad \zeta \in\left(-\infty, \zeta^{-}\right) .
$$


The above two inequalities lead to

$$
\int_{-\infty}^{\zeta^{-}(t)} \frac{\left|\dot{u}_{0} \ddot{a}\left(u_{0}\right)\right| t}{\left(1+\dot{a}\left(u_{0}\right) t\right)^{2}} d \zeta \leq \frac{\left|\dot{u}_{0}\left(\zeta^{-}\right)\right|}{1+\dot{a}\left(u_{0}\left(\zeta^{-}\right)\right) t}=\left|u_{x}(X(t)-0, t)\right| .
$$

This and (5.9) yield the first inequality in (5.7). Using (D2) and (D3) gives

$$
\int_{-\infty}^{\zeta^{-}(t)}\left|\ddot{u}_{0}\right| d \zeta=-\dot{u}_{0}\left(\zeta^{-}\right), \quad \text { for } \quad t \geq T_{1} .
$$

This and (5.5) yield

$$
\int_{-\infty}^{\zeta^{-}(t)} \frac{\left|\ddot{u}_{0}\right|}{1+\dot{a}\left(u_{0}\right) t} d \zeta \leq \frac{\left|\dot{u}_{0}\left(\zeta^{-}\right)\right|}{1+\dot{a}\left(u_{0}\left(\zeta^{-}\right)\right) t}=\left|u_{x}(X(t)-0, t)\right| .
$$

Consequently, the first inequality in (5.8) is proved. The second inequalities in (5.6)-(5.8) can be proved in a similar way.

Let $T=\max \left\{T_{1}, T_{2}\right\}$. Using (5.6)-(5.8) and following the proofs for Lemmas 1.1 and 1.3 , we can show that

$$
\int_{T}^{\infty}\left|u_{x}(X(t) \pm 0, t)\right| d t \leq c, \quad \int_{T}^{\infty}\left\|u_{x x}(\bullet, t)\right\| d t \leq c .
$$

Repeating the proof in Section 3.1, we can obtain

$$
\left\|v_{\epsilon}(\bullet, t)-u(\bullet, t)\right\| \leq\left\|v_{\epsilon}(\bullet, T)-u(\bullet, T)\right\|+c \epsilon, \quad t \in[T, \infty),
$$

where $c$ is independent of $t$ and $\epsilon$. Since the conditions (C1)-(C4) include (D1)(D4), Theorem 4.1 implies that

$$
\left\|v_{\epsilon}(\bullet, t)-u(\bullet, t)\right\| \leq C(T) \epsilon, \quad t \in[0, T] .
$$

Combining (5.11) and (5.12) gives the following theorem.

Theorem 5.1. Let the flux $f$ be strictly convex. Assume that the initial data $u_{0}$ satisfies the requirements (D1)-(D4) stated above. If $v_{\epsilon}$ is the solution to (0.3) and (0.4) and $u$ is the solution to (0.1) and (0.2), then the following error estimate holds:

$$
\sup _{0 \leq t<\infty}\left\|v_{\epsilon}(\bullet, t)-u(\bullet, t)\right\| \leq c \epsilon
$$

where $c$ is a constant independent of $\epsilon$.

\section{Generalizations}

In this section, we will briefly mention two possible generalizations. The first one is a straightforward extension. The ideas in this paper can be applied to a more general viscosity equation of the form

$$
\left(v_{\epsilon}\right)_{t}+f\left(v_{\epsilon}\right)_{x}=\epsilon\left(B\left(v_{\epsilon}\right) v_{\epsilon}\right)_{x x},
$$

where $B(u)>0$ for all $u \in \mathbf{R}$ and $B \in C^{1}(\mathbf{R})$. We only need to change $\Phi$ defined by $(2.7)$ to

$$
\Phi\left(u ; u_{m}^{+}, u_{m}^{-}\right)=\left[f(u)-f\left(u_{m}^{-}\right)-\dot{X}_{m}\left(u-u_{m}^{-}\right)\right] / B(u) .
$$


With this definition of $\Phi$, it can be shown that the corresponding $V_{m}^{(\epsilon)}:=$ $V^{(\epsilon)}\left(x-X_{m}(t) ; u_{m}^{+}, u_{m}^{-}\right)$satisfies

$$
\left(V_{m}^{(\epsilon)}\right)_{t}+f\left(V_{m}^{(\epsilon)}\right)_{x}-\epsilon\left(B\left(V_{m}^{(\epsilon)}\right) V_{m}^{(\epsilon)}\right)_{x x}=\left(V_{m}^{(\epsilon)}\right)_{u_{m}^{+}} \dot{u}_{m}^{+}+\left(V_{m}^{(\epsilon)}\right)_{u_{m}^{-}} \dot{u}_{m}^{-} .
$$

Furthermore, the stability lemma, i.e. Lemma 2.1, can be modified to give an inequality similar to (2.2). It is then straightforward to extend our results and proofs in Sections 3 to 5 to the solution of (6.1).

The second extension is concerned with finite difference approximations for the hyperbolic conservation laws (0.1). We only point out some possible results, and their proofs could be very complicated. As mentioned in the first section that viscosity methods and monotone difference schemes share some common properties when they are applied to approximate solutions to hyperbolic conservation laws, it is expected that Theorem 3.1 can be extended to some monotone difference schemes. More precisely, assuming that $u_{\Delta x}(x, t)$ are solutions of monotone difference schemes consistent with the equation (0.1) and the initial condition (0.2), and assuming that $u_{0}$ satisfies the conditions stated in Theorem 3.1, we expect the following estimate:

$$
\sup _{0 \leq t \leq T}\left\|u_{\Delta x}(\bullet, t)-u(\bullet, t)\right\| \leq C(T)(\Delta x|\log \Delta x|+\Delta x),
$$

where $\Delta x$ is the discrete meshsize in space and $C(T)$ is a constant independent of $\Delta x$. Similar extensions for Theorems 4.1 and 5.1 are also expected to be true. For decreasing piecewise constant initial data, a rigorous analysis, based on Jennings' traveling wave results [8], shows that a uniform error bound similar to that in Theorem 5.1 holds for strictly monotone difference schemes [20]. However, for piecewise smooth solutions, this extension could be quite difficult.

\section{Appendix A.}

Proof of Lemma 2.2. The entropy solution $u$ of (0.1) and (0.2) satisfies $\|u\| \leq$ $\left\|u_{0}\right\|_{L^{\infty}(\mathbf{R})}$. We let

$$
\beta=\max _{|u| \leq\left\|u_{0}\right\|_{L \infty}(\mathbf{R})} f^{\prime \prime}(u) .
$$

From (0.5) and (A.1) we have $\alpha \leq f^{\prime \prime} \leq \beta$. We observe that

$$
\begin{aligned}
& \Phi\left(u ; u_{m}^{+}, u_{m}^{-}\right) \\
= & {\left[\int_{0}^{1} f^{\prime}\left(\theta u+(1-\theta) u_{m}^{-}\right) d \theta-\int_{0}^{1} f^{\prime}\left(\theta u_{m}^{+}+(1-\theta) u_{m}^{-}\right) d \theta\right]\left(u-u_{m}^{-}\right) } \\
= & \int_{0}^{1} f^{\prime \prime}\left(u^{*}\right) \theta d \theta\left(u-u_{m}^{+}\right)\left(u-u_{m}^{-}\right),
\end{aligned}
$$

where $u^{*}$ is some intermediate value. Since $f^{\prime \prime} \geq \alpha>0$, it can be obtained from (2.6), (2.7) and (A.2) that $V\left(-\infty ; u_{m}^{+}, u_{m}^{-}\right)=u_{m}^{-}, V\left(\infty ; u_{m}^{+}, u_{m}^{-}\right)=u_{m}^{+}$. It is easy to see that $V_{\zeta}=\Phi\left(V ; u_{m}^{+}, u_{m}^{-}\right) \leq 0$, which indicates that $V$ is a decreasing function with respect to $\zeta$. From (A.2), we have

$$
\frac{1}{2} \beta\left(u-u_{m}^{+}\right)\left(u-u_{m}^{-}\right) \leq \Phi\left(V ; u_{m}^{+}, u_{m}^{-}\right) \leq \frac{1}{2} \alpha\left(u-u_{m}^{+}\right)\left(u-u_{m}^{-}\right) .
$$


This together with (2.6) and (2.7) imply that for $\zeta \leq 0$ (in this case $\left.V \geq \frac{1}{2}\left(u_{m}^{+}+u_{m}^{-}\right)\right)$

$$
\frac{2}{\alpha} \frac{1}{u_{m}^{-}-u_{m}^{+}} \ln \left[\frac{u_{m}^{-}-V}{V-u_{m}^{+}}\right] \leq \zeta\left(V, u_{m}^{+}, u_{m}^{-}\right) \leq \frac{2}{\beta} \frac{1}{u_{m}^{-}-u_{m}^{+}} \ln \left[\frac{u_{m}^{-}-V}{V-u_{m}^{+}}\right] .
$$

Therefore we have

$$
\tilde{V}\left(\alpha \zeta / 2 ; u_{m}^{+}, u_{m}^{-}\right) \leq V \leq \tilde{V}\left(\beta \zeta / 2 ; u_{m}^{+}, u_{m}^{-}\right), \quad \zeta \leq 0,
$$

where

$$
\tilde{V}\left(\zeta ; u_{m}^{+}, u_{m}^{-}\right)=\frac{u_{m}^{-}+u_{m}^{+} \exp \left\{\zeta\left(u_{m}^{-}-u_{m}^{+}\right)\right\}}{1+\exp \left\{\zeta\left(u_{m}^{-}-u_{m}^{+}\right)\right\}} .
$$

It follows from the above results that, for $\zeta \leq 0$,

$$
\left|V\left(\zeta ; u_{m}^{+}, u_{m}^{-}\right)-u_{m}^{-}\right| \leq\left(u_{m}^{-}-u_{m}^{+}\right) \exp \left\{\alpha \zeta\left(u_{m}^{-}-u_{m}^{+}\right) / 2\right\} .
$$

Similarly, we can show that

$$
\tilde{V}\left(\beta \zeta / 2 ; u_{m}^{+}, u_{m}^{-}\right) \leq V \leq \tilde{V}\left(\alpha \zeta / 2 ; u_{m}^{+}, u_{m}^{-}\right), \quad \zeta \geq 0,
$$

which gives that, for $\zeta \geq 0$,

$$
\left|V\left(\zeta ; u_{m}^{+}, u_{m}^{-}\right)-u_{m}^{+}\right| \leq\left(u_{m}^{-}-u_{m}^{+}\right) \exp \left\{-\alpha \zeta\left(u_{m}^{-}-u_{m}^{+}\right) / 2\right\} .
$$

It follows from (A.4) and (A.5) that

$$
\left\|V\left(\bullet ; u_{m}^{+}, u_{m}^{-}\right)-H\left(\bullet ; u_{m}^{+}, u_{m}^{-}\right)\right\| \leq \frac{4}{\alpha} .
$$

Notice that $H\left(x-X_{m} ; u_{m}^{+}, u_{m}^{-}\right)=H\left(\left(x-X_{m}\right) / \epsilon ; u_{m}^{+}, u_{m}^{-}\right)$. Using integration by substitution and (A.6) gives the inequality (2.10).

Proof of Lemma 2.3. Differentiating (2.6) with respect to the parameter $u_{m}^{+}$gives $0=\frac{V_{u_{m}^{+}}}{\Phi\left(V ; u_{m}^{+}, u_{m}^{-}\right)}-\frac{1}{2} \frac{1}{\Phi\left(\frac{1}{2}\left(u_{m}^{+}+u_{m}^{-}\right) ; u_{m}^{+}, u_{m}^{-}\right)}-\int_{\frac{1}{2}\left(u_{m}^{+}+u_{m}^{-}\right)}^{V} \frac{\left(\Phi\left(u ; u_{m}^{+}, u_{m}^{-}\right)\right)_{u_{m}^{+}}}{\left[\Phi\left(u ; u_{m}^{+}, u_{m}^{-}\right)\right]^{2}} d u$.

We obtain from the above equation that

$$
\begin{aligned}
& V_{u_{m}^{+}}-1=-\frac{1}{2} \frac{\Phi\left(V ; u_{m}^{+}, u_{m}^{-}\right)}{\Phi\left(\frac{1}{2}\left(u_{m}^{+}+u_{m}^{-}\right) ; u_{m}^{+}, u_{m}^{-}\right)} \\
& \quad+\Phi\left(V ; u_{m}^{+}, u_{m}^{-}\right) \int_{\frac{1}{2}\left(u_{m}^{+}+u_{m}^{-}\right)}^{V} \frac{\left(\Phi\left(u ; u_{m}^{+}, u_{m}^{-}\right)\right)_{u_{m}^{+}}+\left(\Phi\left(u ; u_{m}^{+}, u_{m}^{-}\right)\right)_{u}}{\left[\Phi\left(u ; u_{m}^{+}, u_{m}^{-}\right)\right]^{2}} d u .
\end{aligned}
$$

Direct calculation using (2.7) and (2.5) gives

$$
\begin{aligned}
& \Phi_{u_{m}^{+}}+\Phi_{u} \\
= & -\left(\dot{X}_{m}\right)_{u_{m}^{+}}\left(u-u_{m}^{-}\right)+f^{\prime}(u)-\dot{X}_{m} \\
= & -\frac{f^{\prime}\left(u_{m}^{+}\right)\left(u_{m}^{+}-u_{m}^{-}\right)-\left(f\left(u_{m}^{+}\right)-f\left(u_{m}^{-}\right)\right)}{\left(u_{m}^{+}-u_{m}^{-}\right)^{2}}\left(u-u_{m}^{-}\right)+f^{\prime}(u)-\frac{f\left(u_{m}^{+}\right)-f\left(u_{m}^{-}\right)}{u_{m}^{+}-u_{m}^{-}} \\
= & -\frac{f^{\prime}\left(u_{m}^{+}\right)\left(u_{m}^{+}-u_{m}^{-}\right)-\left(f\left(u_{m}^{+}\right)-f\left(u_{m}^{-}\right)\right)}{\left(u_{m}^{+}-u_{m}^{-}\right)^{2}}\left(u-u_{m}^{+}\right)+f^{\prime}(u)-f^{\prime}\left(u_{m}^{+}\right) \\
= & -\frac{f^{\prime \prime}\left(\zeta_{1}\right)}{2}\left(u-u_{m}^{+}\right)+f^{\prime \prime}\left(\zeta_{2}\right)\left(u-u_{m}^{+}\right),
\end{aligned}
$$


where $u_{m}^{+}<\zeta_{1}, \zeta_{2}<u_{m}^{-}$. Therefore, we have obtained that $\left|\Phi_{u_{m}^{+}}+\Phi_{u}\right| \leq \beta\left|u-u_{m}^{+}\right|$, where $\beta$ is given in (A.1). For $\zeta \geq 0$, we have $u_{m}^{+} \leq V \leq \frac{1}{2}\left(u_{m}^{+}+u_{m}^{-}\right)$. From (A.7) and (A.3), we have

$$
\begin{aligned}
& \left|V_{u_{m}^{+}}-1\right| \\
\leq & C \frac{\left|V-u_{m}^{+}\right|}{u_{m}^{-}-u_{m}^{+}}+C\left|V-u_{m}^{+}\right|\left(u_{m}^{-}-u_{m}^{+}\right) \int_{\frac{1}{2}\left(u_{m}^{+}+u_{m}^{-}\right)}^{V} \frac{1}{\left|u-u_{m}^{+}\right|\left(u_{m}^{-}-u_{m}^{+}\right)^{2}} d u \\
\leq & C \frac{\left|V-u_{m}^{+}\right|}{u_{m}^{-}-u_{m}^{+}}\left(1+\ln \left(\frac{\frac{1}{2}\left(u_{m}^{-}-u_{m}^{+}\right)}{V-u_{m}^{+}}\right)\right),
\end{aligned}
$$

where $C$ is some constant depending only on $\alpha$ and $\beta$. Since $x(1+\ln (A / x))$ is an increasing function for $0<x \leq A$, we obtain from (2.9) that

$$
\begin{aligned}
& \int_{0}^{\infty}\left|V_{u_{m}^{+}}\left(\zeta ; u_{m}^{+}, u_{m}^{-}\right)-1\right| d \zeta \\
\leq & C \int_{0}^{\infty} e^{-\left(u_{m}^{-}-u_{m}^{+}\right) \zeta / 2}\left[1+\ln \left(\frac{1}{2} e^{\left(u_{m}^{-}-u_{m}^{+}\right) \zeta / 2}\right)\right] d \zeta \leq \frac{C}{u_{m}^{-}-u_{m}^{+}} .
\end{aligned}
$$

In a similar way we obtain that

$$
V_{u_{m}^{-}}=\frac{1}{2} \frac{\Phi\left(V ; u_{m}^{+}, u_{m}^{-}\right)}{\Phi\left(\frac{1}{2}\left(u_{m}^{+}+u_{m}^{-}\right) ; u_{m}^{+}, u_{m}^{-}\right)}+\Phi\left(V ; u_{m}^{+}, u_{m}^{-}\right) \int_{\frac{1}{2}\left(u_{m}^{+}+u_{m}^{-}\right)}^{V} \frac{\left(\Phi\left(u ; u_{m}^{+}, u_{m}^{-}\right)\right)_{u_{m}^{-}}}{\left[\Phi\left(u ; u_{m}^{+}, u_{m}^{-}\right)\right]^{2}} d u
$$

which also gives

$$
\int_{0}^{\infty}\left|V_{u_{m}^{-}}\left(\zeta ; u_{m}^{+}, u_{m}^{-}\right)\right| d \zeta \leq \frac{C}{u_{m}^{-}-u_{m}^{+}} .
$$

Combining the above two inequalities yields

$$
\begin{aligned}
& \int_{0}^{\infty}\left|\left(V^{(\epsilon)}\right)_{u_{m}^{+}}\left(x ; u_{m}^{+}, u_{m}^{-}\right) \dot{u}_{m}^{+}+\left(V^{(\epsilon)}\right)_{u_{m}^{-}}\left(x ; u_{m}^{+}, u_{m}^{-}\right) \dot{u}_{m}^{-}-H\left(x ; \dot{u}_{m}^{+}, \dot{u}_{m}^{-}\right)\right| d x \\
& =\epsilon \int_{0}^{\infty}\left|V_{u_{m}^{+}}\left(\zeta ; u_{m}^{+}, u_{m}^{-}\right) \dot{u}_{m}^{+}+V_{u_{m}^{-}}\left(\zeta ; u_{m}^{+}, u_{m}^{-}\right) \dot{u}_{m}^{-}-H\left(\zeta ; \dot{u}_{m}^{+}, \dot{u}_{m}^{-}\right)\right| d \zeta \\
& \leq C \epsilon \frac{\left|\dot{u}_{m}^{+}\right|+\left|\dot{u}_{m}^{-}\right|}{u_{m}^{-}-u_{m}^{+}}
\end{aligned}
$$

where we have used the change of variable $\zeta=x / \epsilon$. It can be verified that

$$
\left|\dot{u}_{m}^{ \pm}\right|=\left|\left(\dot{X}_{m}-a\left(u_{m}^{ \pm}\right)\right) u_{x}\left(X_{m} \pm 0, t\right)\right| \leq C\left|u_{m}^{+}-u_{m}^{-}\right|\left|u_{x}\left(X_{m} \pm 0, t\right)\right| .
$$

The above results lead to

$$
\begin{aligned}
& \int_{0}^{\infty}\left|\left(V^{(\epsilon)}\right)_{u_{m}^{+}}\left(x ; u_{m}^{+}, u_{m}^{-}\right) \dot{u}_{m}^{+}+\left(V^{(\epsilon)}\right)_{u_{m}^{-}}\left(x ; u_{m}^{+}, u_{m}^{-}\right) \dot{u}_{m}^{-}-H\left(x ; \dot{u}_{m}^{+}, \dot{u}_{m}^{-}\right)\right| d x \\
& \leq C \epsilon\left(\left|u_{x}\left(X_{m}+0, t\right)\right|+\left|u_{x}\left(X_{m}-0, t\right)\right|\right) .
\end{aligned}
$$

A similar estimate holds for the integral with the same integrand over $(-\infty, 0)$. This completes the proof of the lemma.

\section{ACKNOWLEDGEMENT}

We thank the referees for helpful comments and suggestions. 


\section{REFERENCES}

[1] N. S. Bakhvalov, Estimation of the error of numerical integration of a first-order quasilinear equation, Zh. Vychisl. Mat. i Mat. Fiz., 1 (1961), pp. 771-783; English transl. in USSR Comput. Math. and Math. Phys., 1 (1962), pp. 926-938. MR 27:2123

[2] C. M. Dafermos, Generalized characteristics and the structure of solutions of hyperbolic conservation laws, Indiana Univ. Math. J., 26 (1977), pp. 1097-1119. MR 56:16151

[3] H. Fan, Existence of discrete traveling waves and error estimates for Godunov schemes of conservation laws, Preprint (1996).

[4] J. Goodman and Z. Xin, Viscous limits for piecewise smooth solutions to systems of conservation laws, Arch. Rational Mech. Anal., 121 (1992), pp. 235-265. MR 93k:35167

[5] E. Harabetian, Rarefactions and large time behavior for parabolic equations and monotone schemes, Comm. Math. Phys., 114 (1988), pp. 527-536. MR 89d:35084

[6] A. Harten, The artificial compression method for computation of shocks and contact discontinuities, Comm. Pure Appl. Math., 30 (1977), pp. 611-638. MR 55:11637

[7] A. Harten, J. M. Hyman and P. D. Lax, On finite difference approximations and entropy conditions for shocks, Comm. Pure Appl. Math., 29 (1976), pp. 297-322. MR 54:1640

[8] G. Jennings, Discrete shocks, Comm. Pure Appl. Math. 27 (1974), pp. 25-37. MR 49:3358

[9] H.-O. Kreiss and J. Lorenz, Initial-Boundary Value Problems and the Navier-Stokes Equations, Academic Press, 1989. MR 91a:35138

[10] N. N. Kuznetsov, Accuracy of some approximate methods for computing the weak solutions of a first-order quasi-linear equation, USSR Comput. Math. and Math. Phys., 16 (1976), pp. 105-119. MR 58:3510

[11] P. D. Lax, Hyperbolic systems of conservation laws II, Comm. Pure Appl. Math., 10 (1957), pp. 537-566. MR 20:176

[12] J. Liu and Z. Xin, $L^{1}$-stability of stationary discrete shocks, Math. Comp., 60 (1993), pp. 233-244. MR 93d:35097

[13] R. J. LeVeque, Numerical Methods for Conservation Laws, Birkhauser, 2nd ed., 1992. MR 92m:65106

[14] B. J. Lucier, Error bounds for the methods of Glimm, Godunov and LeVeque, SIAM J. Numer. Anal., 22 (1985), pp. 1074-1081. MR 88a:65104

[15] O. A. Oleinik, Discontinuous solutions of non-linear differential equations, Amer. Math. Soc. Transl., 26 (1963), pp. 95-172. MR 27:1721

[16] J. Smoller, Shock Waves and Reaction-Diffusion Equations, Springer-Verlag, 1983. MR 84d:35002

[17] D. G. Schaeffer, A regularity theorem for conservation laws, Adv. in Math., 11 (1973), pp. 368-386. MR 48:4523

[18] E. Tadmor and T. Tassa, On the piecewise smoothness of entropy solutions to scalar conservation laws, Commun. in PDEs, 18 (1993), pp. 1631-1652. MR 94g:35144

[19] T. Tang and Z.-H. Teng, The sharpness of Kuznetsov's $O(\sqrt{\Delta x}) L^{1}$-error estimate for monotone difference schemes. Math. Comp., 64 (1995), pp.581-589. MR 95f:65176

[20] Z.-H. Teng and P. W. Zhang, Optimal $L^{1}$-rate of convergence for viscosity method and monotone scheme to piecewise constant solutions with shocks, 1994. To appear in SIAM J. Numer. Anal.

Department of Mathematics and Statistics, Simon Fraser University, Burnaby, British Columbia, Canada V5A 1S6

E-mail address: ttang@sfu.ca

Department of Mathematics, Peking University, Beijing 100871, China

E-mail address: tengzh@sxx0.math.pku.edu.cn 